\title{
Provenance analyses of the heavy-mineral beach sands of the Annaba coast, northeast Algeria, and their consequences for the evaluation of fossil placer deposit
}

\author{
Asma Chemam ${ }^{1, *}$, Soraya Hadjzobir ${ }^{2}$, Menana Daif $^{1}$, Uwe Altenberger ${ }^{3}$ \\ and Christina GüNTER ${ }^{3}$ \\ ${ }^{1}$ Laboratory of LGRN, Badji-Mokhtar/Annaba University, P.O. Box 12, 23000 Annaba, Algeria. \\ ${ }^{2}$ Laboratory of Soils and Sustainable Development, Badji-Mokhtar/Annaba University, P.O. Box 12, \\ 23000 Annaba, Algeria. \\ ${ }^{3}$ Institute of Earth and Environmental Science, University of Potsdam, Karl-Liebknecht-Strasse 24-25, \\ D14476 Potsdam-Golm, Germany. \\ *Corresponding author. e-mail: Chemam.asma@gmail.com
}

MS received 10 April 2017; revised 13 December 2017; accepted 12 March 2018; published online 26 October 2018

The paper presents the first study of heavy-mineral sand beaches from the Mediterranean coast of Annaba/Algeria. The studied beaches run along the basement outcrops of the Edough massif, which are mainly composed by micaschists, tourmaline-rich quartzo-feldspathic veins, gneisses, skarns and marbles. Sand samples were taken from three localities (Ain Achir, Plage-Militaire and El Nasr). The heavy-mineral fraction comprises between 74 and 91 vol\%. The garnets of the beaches are almandine rich and tourmalines vary with respect to their location from schorl to dravite. Tourmaline at Ain Achir and the Plage-Militaire is schorlits, while at El Nasr beach dravite is ubiquitous. The World Shale Average normalised REE of the sands and the basement outcrops reveal: (i) Ain Achir beach: REE pattern of sand and the coastal rocks from the studied beaches reflects a multiple sources; (ii) Plage-Militaire: the sand and the coastal outcrops show similar LREE and a strong enrichment in HREE, suggesting the presence HREE-rich phases found as inclusions in staurolite; (iii) El Nasr: two types of sand patterns are found: one with flat REE pattern similar to the proximal rocks and other one enriched in HREE suggesting a mixed source.

Keywords. Provenance; heavy minerals; beach sediments; fossil placer; geochemistry; Annaba/Algeria.

\section{Introduction}

The mineralogy of modern beaches depends on the nature of a source rock from which the detrital material is derived, and comprises minerals that are resistant to weathering. Heavy-sand components are mainly derived through erosive processes on rock outcrops. The studied area consists of three flat sandy coast beaches in front of the
Edough massif (Annaba/Algeria). Out of dozen, only three beaches show high concentrations of toned heavy-mineral sands: the Ain Achir beach characterised by light- and dark-toned sands and the Plage-Militaire and El Nasr beaches comprising consistently dark-toned sands. The outcrops surrounding these three beaches are garnet-staurolite (and/or andalusite), and kyanite micaschists, marbles, epidotic skarns, migmatites and numerous 
tourmaline-bearing quartzo-feldspathic veins. This paper presents the first study of the provenance of dark-toned heavy-mineral sands from coastlines of Annaba/Algeria and of Algeria.

\section{Study area}

\subsection{Actual geographic setting}

The studied areas are located at the northeastern coast of Annaba city (figure 1a). They are composed of two fields: the Cap de Garde field and the Harbor field. The Cap de Garde field (figure 1b) is composed of two small beaches: Ain Achir $(275 \times 30 \mathrm{~m})$ and Plage-Militaire $(236 \times 20 \mathrm{~m})$, their shoreline lay between $36^{\circ} 57^{\prime} 52^{\prime \prime}-36^{\circ} 57^{\prime} 51^{\prime \prime} \mathrm{N}$ latitude and $7^{\circ} 46^{\prime} 89^{\prime \prime}-7^{\circ} 46^{\prime} 94^{\prime \prime} \mathrm{E}$ longitude, respectively. The Harbor field (figure 1c) includes the El Nasr beach, its shoreline is between $36^{\circ} 54^{\prime} 36^{\prime \prime}-$ $36^{\circ} 54^{\prime} 31^{\prime \prime} \mathrm{N}$ and $7^{\circ} 46^{\prime} 17-7^{\circ} 46^{\prime} 24^{\prime \prime} \mathrm{E}$.

\subsubsection{Temperature and rainfall}

Annaba is characterised by a warm-temperature climate. The climate map of Köppen-Geiger (Hufty 2001) classifies the climate as Csa (warm temperature to dry climate). Annaba displays an

(a)
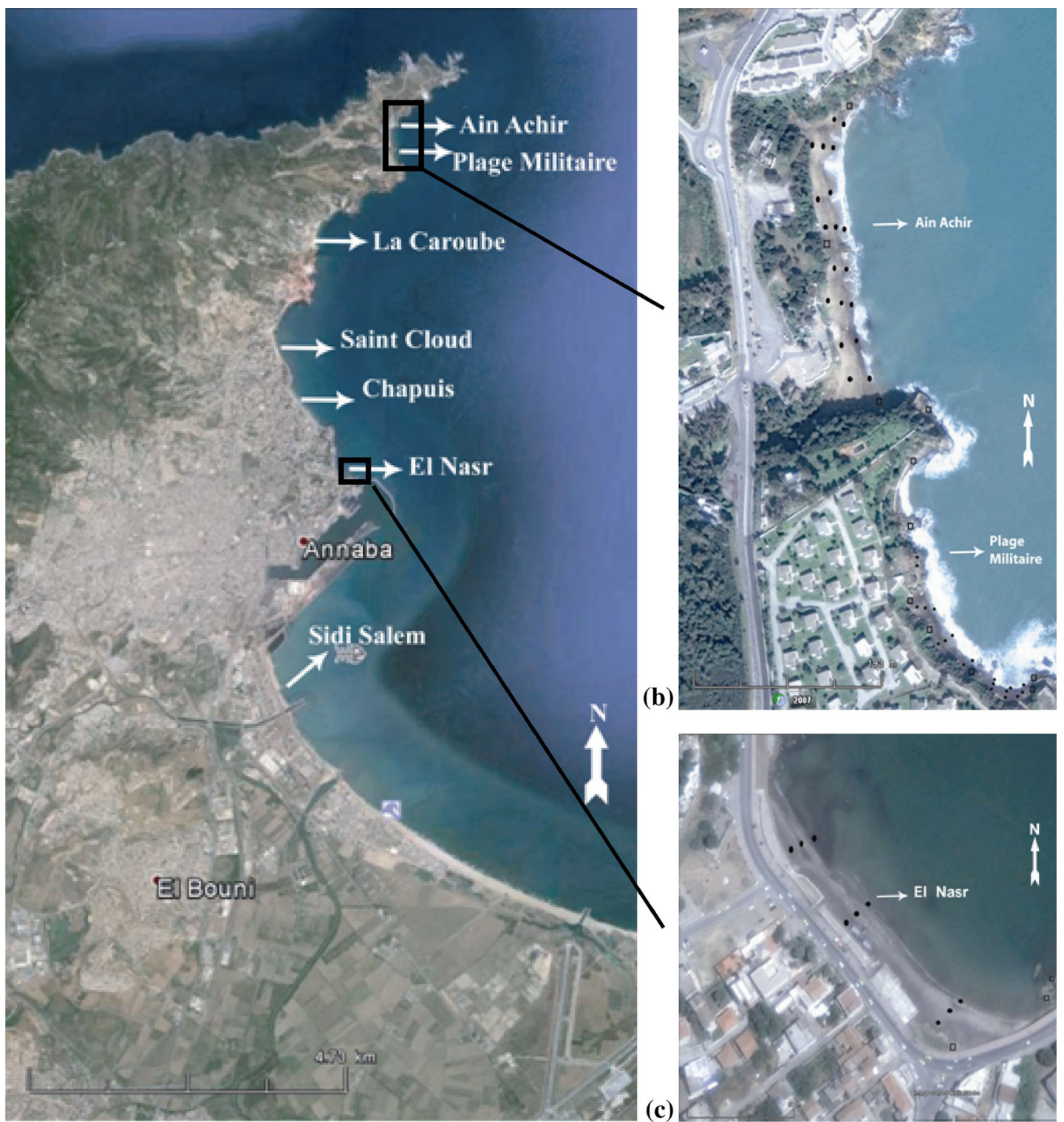

Figure 1. Aerial photographs: (a) the Annaba town coast, (b) the Cap de Garde field with the two studied beaches (Ain Achir and the Plage-Militaire beaches), and (c) the Harbor field (El Nasr beach). $\bullet=$ sand samples, $\square=$ proximal rock outcrops samples locations. Google Earth 2016. 
average temperature of $18.4^{\circ} \mathrm{C}$ throughout the year. Rainfall averages $712 \mathrm{~mm}$ (650 and 1000 $\mathrm{mm} / \mathrm{yr}$; ANDI 2013) per year. The probability of rainfall is very high during the period JanuaryMarch and during October-December (temperatureweather.com 2016).

\subsubsection{Hydrography}

The hydrographic network of the Annaba region is rather dense (Hilly 1962; figure 2a). It consists mainly of Lake Fetzara (freshwater) covering 18,670 ha and the Oued Seybouse with a length of $127.5 \mathrm{~km}$ (ANDI 2013).

\subsubsection{Topography}

The relief of the Annaba region is dominated by the Edough massif ( $52 \%$ ), and shaped by the hogbacks $(28 \%)$ and the plains $(18 \%)$ as well. The Edough massif extends over a length of $50 \mathrm{~km}$ in the direction SW-NE. The central part of the massif culminates at $1008 \mathrm{~m}$ (Bouazizi). The altitudes of the southwestern (Bou-Maiza) and northeastern (Cap de Garde) slopes are low, on average 200 $300 \mathrm{~m}$ (Oularbi and Zeghiche 2009). Depending on the intensity of erosion, Oularbi and Zeghiche (2009) distinguish four principal erosional zones in the Edough massif (figure $2 \mathrm{~b}$ ), i.e., zones with: (i) intense erosion, (ii) medium erosion, (iii) low

(a)

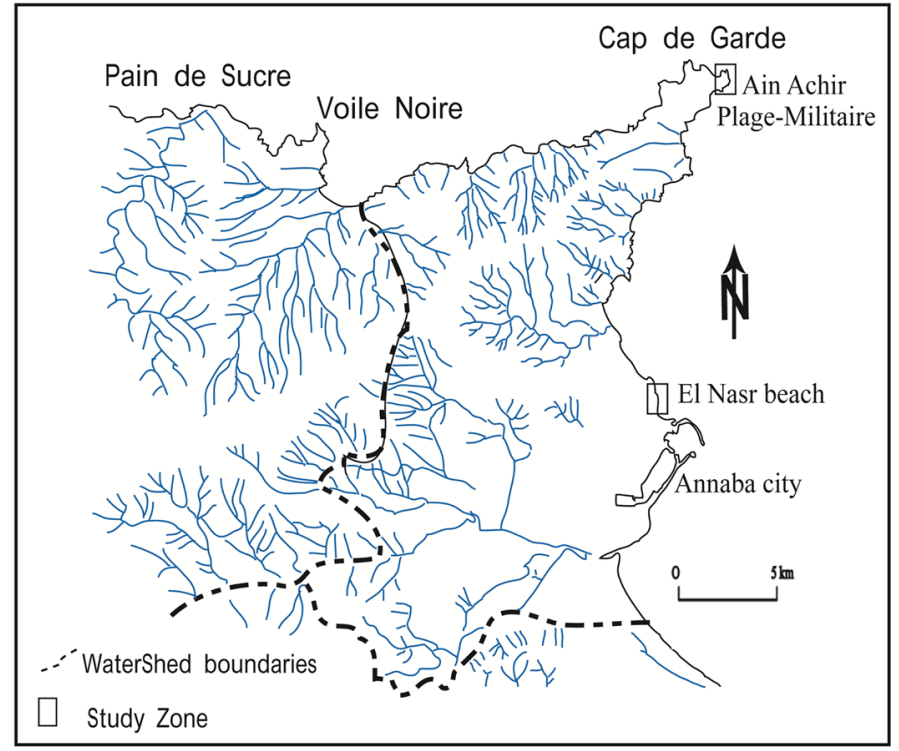

(b)

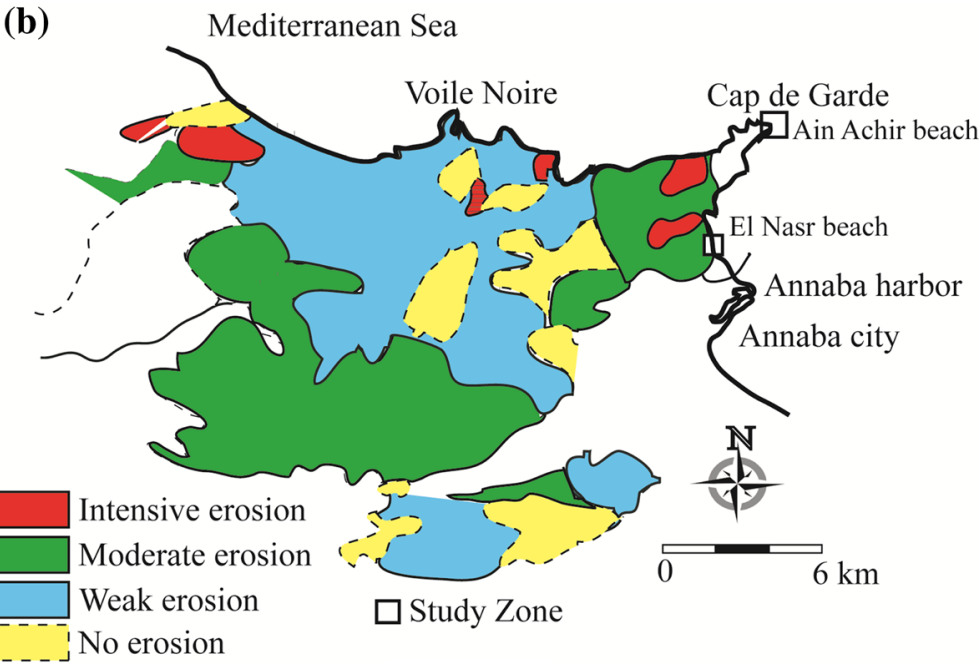

Figure 2. Maps of (a) the hydrographic network of the Annaba region (Hilly 1962), modified, and (b) erosion susceptibility of the Edough massif (Oularbi and Zeghiche 2009). 
erosion and (iv) zero erosion. The erosional sensitivity map (Oularbi and Zeghiche 2009) shows that the study areas (Ain Achir, the Plage-Militaire and El Nasr beaches) lay in the 'zero erosion' zone.

\subsubsection{Swell and sea wave}

The coasts of Annaba are characterised by a north-northwestern swell. In winters, the height of the waves is high and can exceed $3 \mathrm{~m}$. In summer, the height of the waves is low and does not exceed $1 \mathrm{~m}$.

\subsubsection{Morphology}

The geomorphology of the study area is characterised by a flat topography and the lack of an active hydrographic system, the Cap de Garde (Ain Achir and the Plage-Militaire beach) and the Harbor (El Nasr beach) fields are characterised by low continental erosion.

\subsection{Geological background}

Geologically, the studied areas are a part of the crystalline Edough Massif. The latter experienced a regional and polycyclic metamorphism characterised by high-pressure (12-14 kbar) and mediumtemperature metamorphic conditions $\left(500-600^{\circ} \mathrm{C}\right)$ (Brunnel et al. 1988; Caby and Hammor 1992; Ahmed-Said et al. 1993; Caby et al. 2001). The different metamorphic units underwent a first oblique deformation characterised by syn-metamorphic folds followed by flexural shear generating upright folds of $140^{\circ} \mathrm{N}$ direction, anticlines with direction $50-60^{\circ} \mathrm{N}$ and shear senses of $120-160^{\circ} \mathrm{N}$. In the studied areas, the coastal rock outcrops are mainly composed by garnet, staurolite (and/or andalusite), kyanite micaschists, marble, epidotic skarns, migmatites and numerous quartzofeldspathic veins (figure 3).

\subsubsection{Cap de Garde field}

The two beaches at this field are underlain by metamorphic rocks. The northern part of the Cap de Garde is composed by kyanite-bearing micaschists (figure 3a), migmatised gneisses (figure 3b) followed by tourmaline-bearing quartzo-feldspathic veins occurring in garnet-staurolite micaschists and/or in garnet micaschists (figure 3c) and small garnetite outcrops (figure 3d). In these beaches, most of the outcrops are staurolite-garnet micaschists with some quartz veins (figure 3e) or staurolite-garnet micaschist alternating with marble layers. The contact between the staurolitegarnet micaschists and the marble layers is marked by epidote-rich skarns (figure 3f). The northern part of the Plage-Militaire beach is composed by garnet micaschists with some tourmaline-rich quartzo-feldspathic veins (figure $3 \mathrm{~g}$ ) and the same garnet-staurolite micaschist alternating with marble layers as at the Ain Achir beach. The other parts show mainly staurolite-garnet micaschists (figure $3 \mathrm{~h}$ ).

\subsubsection{The Harbor field}

This field encloses the El Nasr beach. Concrete slabs cover the back of the shoreline. Only a 20-m garnet-staurolite micaschist outcrop with tourmaline-rich veins is visible in the southern part of the beach (figure $3 i$ and $j$ ).

\section{Analytical methods and sampling}

Sands from the studied beaches and the proximal rock outcrops have been sampled. A set of 23 handspecimen rock samples (8 at Ain Achir, 12 at the Plage-Militaire and 3 at El Nasr beach) and 44 (18 at Ain Achir, 17 at the Plage-Militaire and 9 at El Nasr beach) surface sand samples with a single weight of $3 \mathrm{~kg}$ were taken. The sand sampling was done along with a $10 \times 15 \mathrm{~m}$ surface grid and a depth of 25-30 cm. The bulk sample was reduced by coning and quartering and a $100 \mathrm{~g}$ portion of the sample was selected for the analysis (following Darnley et al. 1995). Powders of the selected unaltered samples were dried overnight at $105^{\circ} \mathrm{C}$ prior to the analyses. The compositions of the whole-rock samples, including major, minor and trace elements, were determined by wavelength dispersive X-ray fluorescence spectrometry, inductively coupled plasma-mass spectrometry and inductively coupled plasma-atomic emission spectrometry at laboratories of the GeoForschungsZentrum Potsdam and the Institute of Earth and Environmental Science, Potsdam University, Germany. International and internal reference samples were used for calibration. Analyses for major and minor elements and for trace elements were conducted on fused glass and pressed powder disks, respectively. Both of the disks were prepared at the Institute of Earth and Environmental Science, Potsdam. 
(d)

(a)
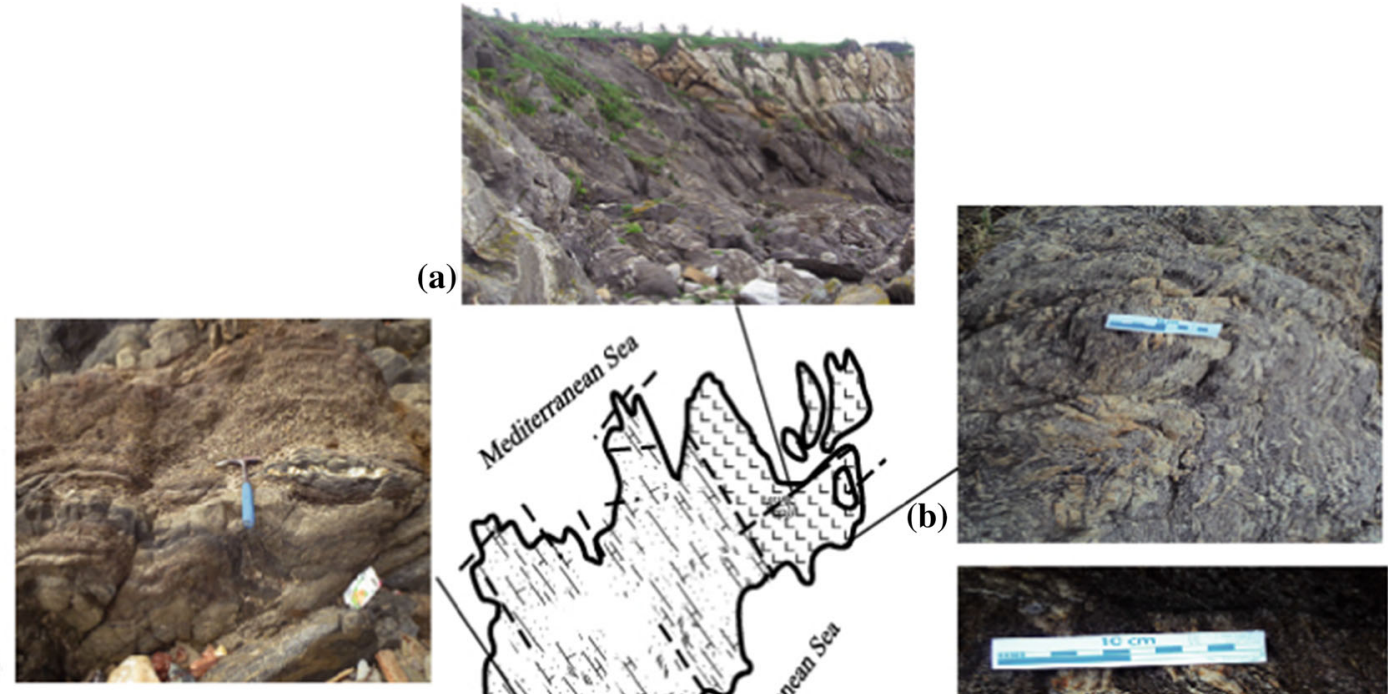

(e)
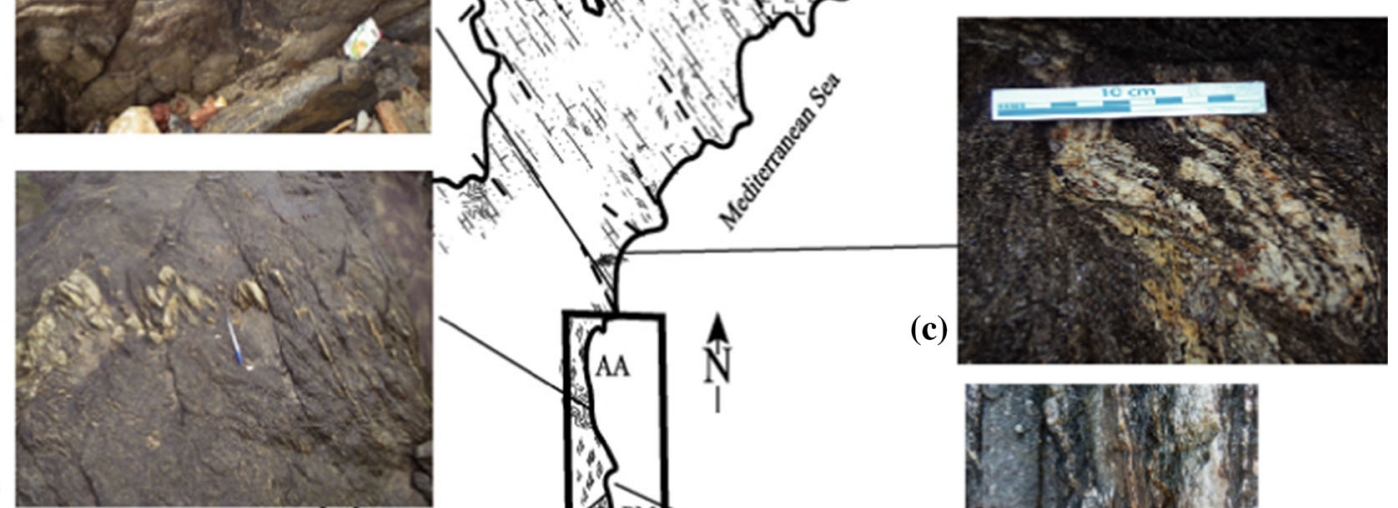

(f)

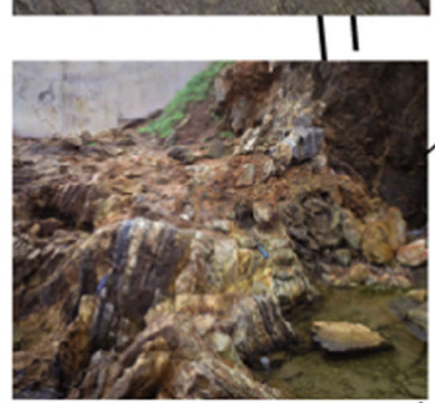

(h)
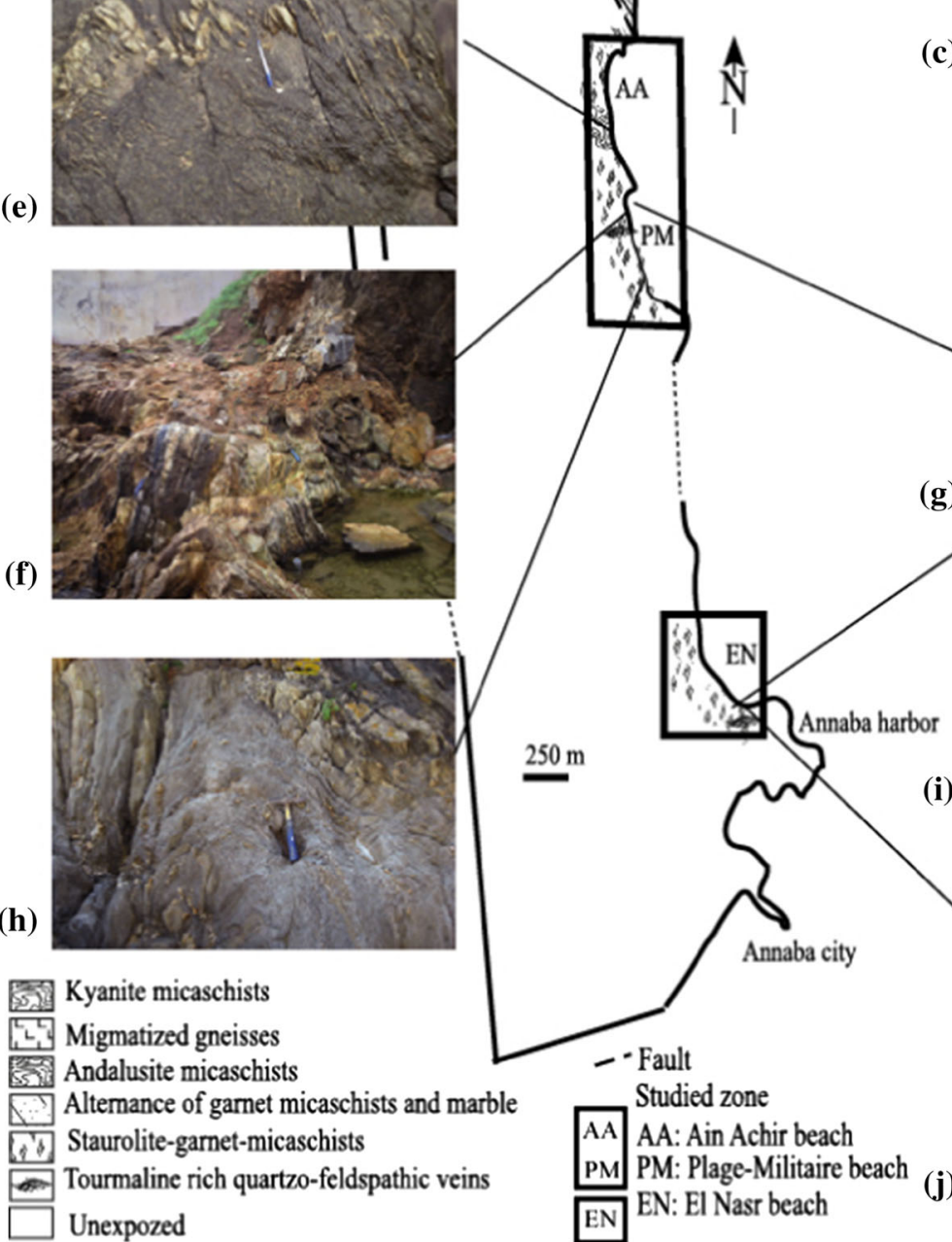

(g)

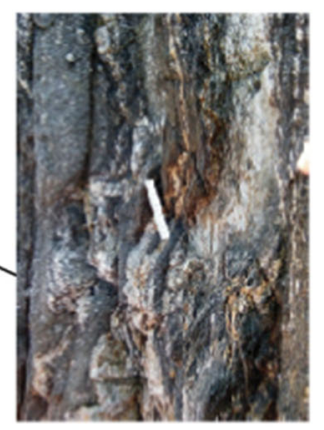

(i)
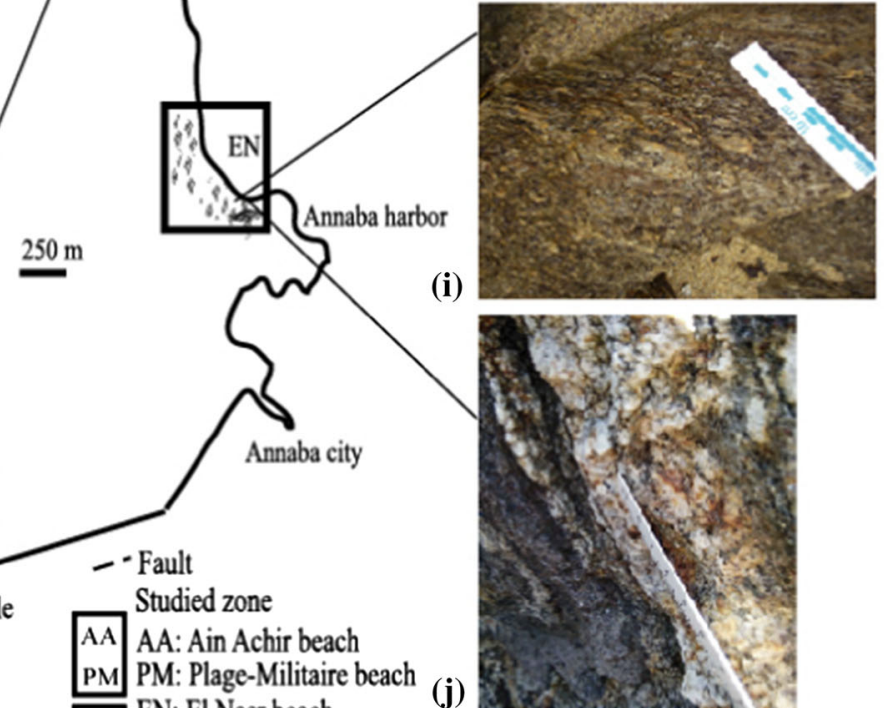

EN EN: El Nasr beach

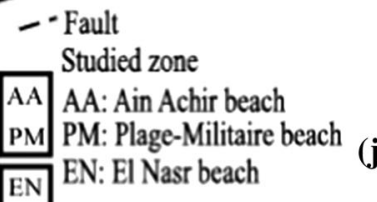

Figure 3. Simplified geological maps of the Cap de Garde (AA-PM) and the Harbor (EN) fields with the studied beach locations and field photography of the basement outcrops: (a) kyanite-bearing micaschist, (b) migmatised gneisses, (c) tourmaline-bearing quartzo-feldspathic veins, (d) small garnetite outcrops, (e) staurolite-garnet micaschist with quartz veins, (f) skarns, (g) garnet micaschist with tourmaline-rich quartzo-feldspathic layers, (h) staurolite-garnet micaschists, (i) garnet-staurolite micaschist, and (j) micaschist with tourmaline-rich quartzo-feldspathic veins. 
Whole heavy-mineral grains were identified under incident light using a binocular microscope. Random grain samples were also mounted in thin section for identification using a petrographic microscope, thus providing a means of verifying the bulk identifications achieved under incident light. The minerals identified by microscopic investigations were further checked by X-ray.

The mineral chemical analyses were conducted using a fully automated JEOL JXA-8200 electron microprobe at the Institute of Earth and Environmental Science laboratory/Potsdam.

At least 100 dark-toned minerals were counted for mode composition, using the ribbon counting method (Mange and Maurer 1991; Morton and Hallsworth 1994). Counts of minerals were made using microphotographs taken along regular grid spacing on the thin section and using the free software Mesurim.

\section{Results}

\subsection{Petrography and mineralogy}

\subsubsection{Proximal belts outcrops}

Cap de Garde field (Ain Achir beach): Three samples representing the major proximal outcrops of the Ain Achir beach have been studied under a petrographic microscope. The samples (E60, E62 and E74) are micaschists. Kyanite-bearing micaschists at northern part of the Cap de Garde (Vivier zone) have also been sampled (five samples Viv1-5 and Dviv). Samples of the micaschists are mainly composed by elongated and sometimes cracked staurolite with zircon inclusions, altered biotite, quartz with wavy extinction and euhedral garnet with inclusions (figure $4 \mathrm{a}$ and $\mathrm{b}$ ). The mineralogical composition of the skarn sample is calcite, amphibole, epidote and rare scapolite (figure 4c and d). Kyanite from the Vivier micaschists is elongated with minor quartz inclusions.

Plage-Militaire beach: The outcrop is composed of a garnet-staurolite with rare kyanite micaschists. The staurolite grains are smaller than those of the Ain Achir field. Kyanite grains elongated with minor garnet inclusions (figure 4e). Garnets are euhedral and show sometimes quartz inclusions (figure 4f).

Harbor field(El Nasr beach): The basement outcrop is very small $(5 \mathrm{~m})$, and composed of a garnetstaurolite micaschists. Feldspars are highly altered (figure 4g). Small garnets are common (figure 4h).

\subsubsection{Sand description}

The Ain Achir and the Plage-Militaire sand show significant differences in their mode (table 1). The Ain Achir sand shows two kinds of colours: light coloured sands, containing an average of $39 \%$ heavy minerals, and dark sands, showing an average of $74 \%$ heavy minerals. The black sand typically consists of staurolite (43\%) as the main mineral, followed by garnet (19\%), tourmaline (9\%), kyanite and epidote $(2 \%)$. The Plage-Militaire is characterised by a higher average in heavy-mineral content (91\%). As in the Ain Achir sand, staurolite is the main mineral (45\%) followed by garnet $(29 \%)$. The tourmaline content is higher (13\%) than in Ain Achir sand, whereas kyanite and epidote are low (2 and 1\%, respectively).

The binocular analysis of the Ain Achir and Plage-Militaire dark sand yielded a variegated spectrum of minerals (figure 5) with dominantly metastable minerals: (i) epidote occurs in both the beach sand samples in relatively low abundance ranging from 1 to $2 \%$. Under the stereo-microscope, epidote is pale green with short prism and sometimes rounded shape (figure 5a), (ii) staurolite is well represented and is the most common mineral in the two beaches. The crystals have dark yellowish brown colour. They occur as irregular, elongated or fractured grains. Some crystals show zircon inclusions (figure $5 \mathrm{~b}$ and $\mathrm{c}$ ), (iii) garnets occur as euhedral, subrounded to rounded crystals (figure 5a and b). Some grains are sharp irregular fragments. All crystals are pale pink to red. Some are pinkish brown, ilmenite and quartz inclusions are common, but some garnets from the Plage-Militaire sand show also zircon inclusions (not shown), (iv) kyanite is present in prismatic fragments. All crystals are sharp edged. Some crystals show small typical kyanite cleavage and exhibit characteristic step-like fractures, ilmenite inclusions are common (figure $5 \mathrm{~b}$ and $\mathrm{c})$.

There are no major differences between the mineralogical composition of the El Nasr sand and those from the Cap de Garde. The El Nasr sand is characterised by an average of $84 \%$ of heavy mineral (staurolite $60 \%$, garnet $14 \%$, kyanite $5 \%$, tourmaline $4 \%$ ) and an average of light minerals $(16 \%)$ composed of quartz and calcite (table 2). The heavy-mineral assemblage is restricted to few selective minerals such as: pink garnet with quartz and/or ilmenite inclusions, yellow-brown staurolite with inclusions (figure $5 \mathrm{~d}$ and e) and kyanite in 
(a)

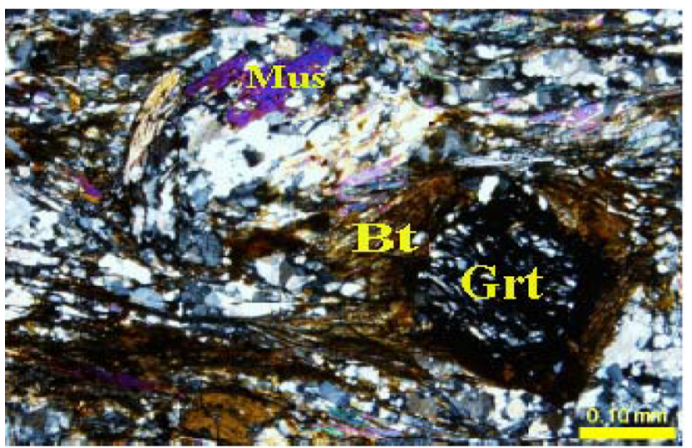

(c)

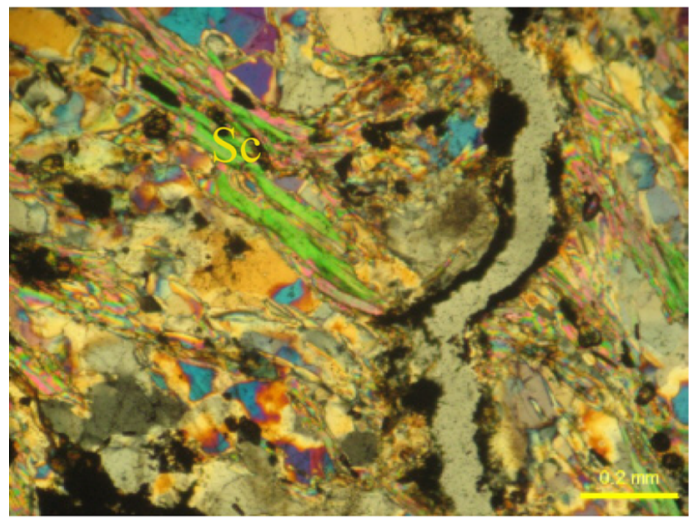

(e)

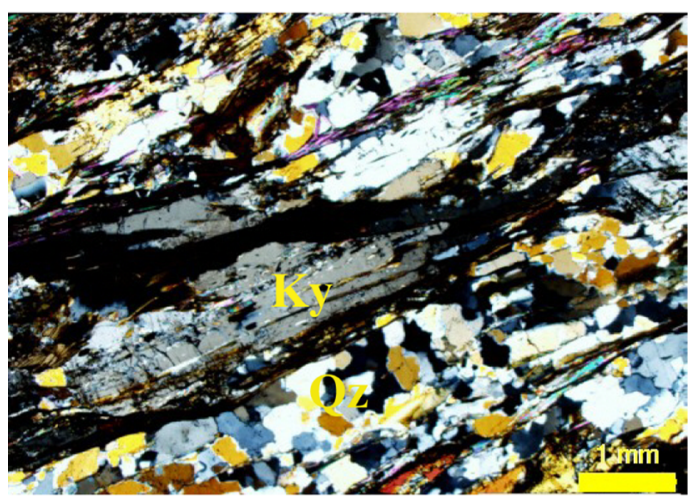

(g)

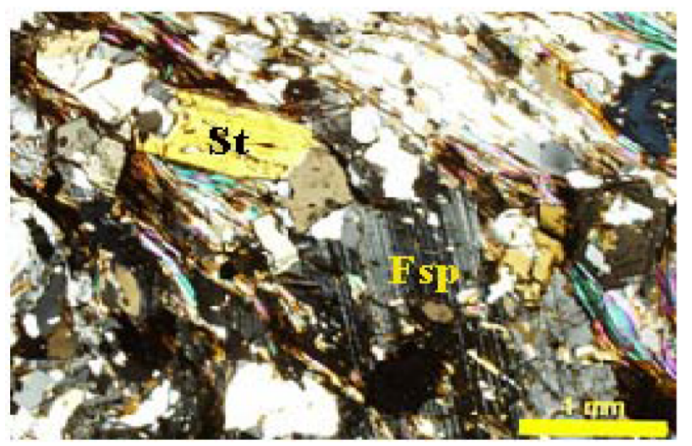

(b)

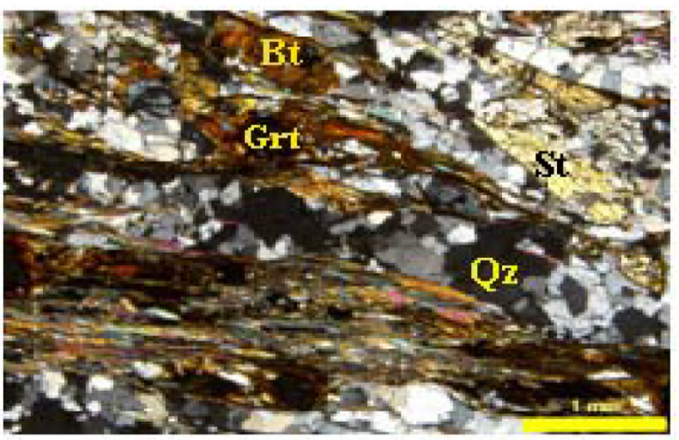

(d)

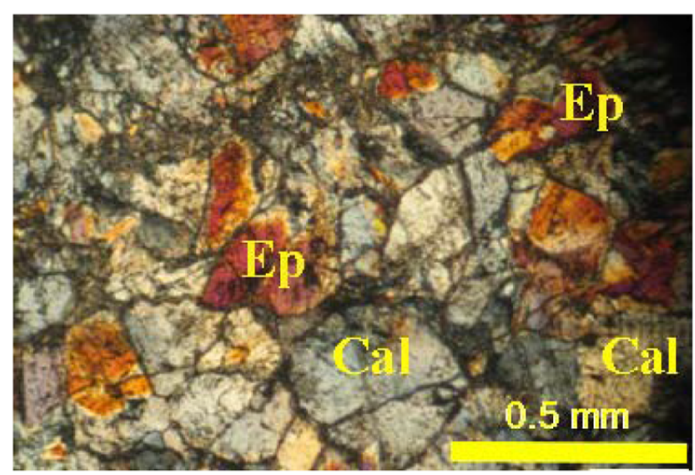

(f)

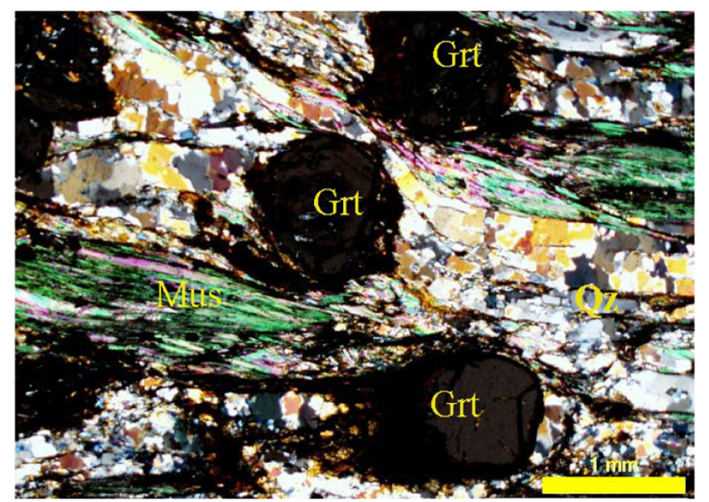

(h)

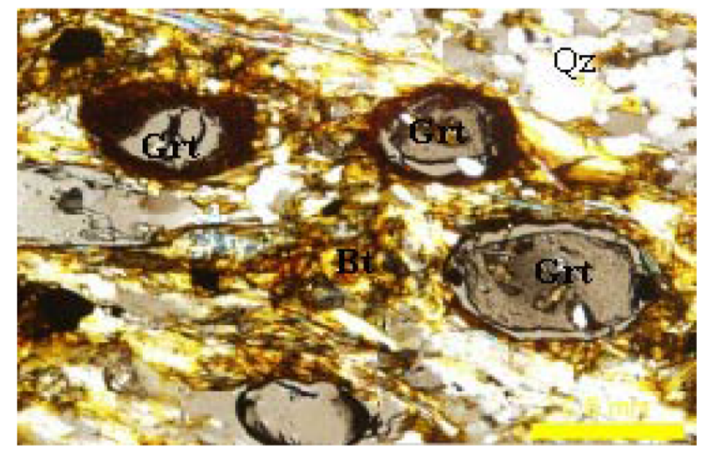

Figure 4. Microphotograph of proximal outcrops of the studied fields: Ain Achir: (a and b) micaschist, (c and d) epidoterich skarn; Plage-Militaire: (e) garnet-staurolite-kyanite micaschist, and (f) garnet micaschist; El Nasr: (g) garnet-staurolite micaschist with some feldspars, and (h) garnet micaschist. 
Table 1. Heavy-mineral composition of the sands from Ain Achir, Plage-Militaire and El Nasr beaches.

\begin{tabular}{|c|c|c|c|c|c|c|c|c|c|c|}
\hline \multicolumn{2}{|l|}{ Samples } & Grt & $\mathrm{St}$ & Ky & Tur & Ep & $\mathrm{Qz}$ & Cal & $\Sigma \mathrm{HM} \%$ & $\Sigma L M \%$ \\
\hline \multicolumn{11}{|c|}{ Ain Achir beach (AA) } \\
\hline \multirow[t]{8}{*}{ Light sand (LS) } & AA1a & 4 & 10 & 4 & 8 & 9 & 7 & 57 & 36 & 64 \\
\hline & AA1b & 7 & 36 & 5 & 4 & 0 & 10 & 38 & 52 & 48 \\
\hline & $\mathrm{AA} 2 \mathrm{~b}$ & 5 & 10 & 4 & 4 & 3 & 14 & 59 & 27 & 73 \\
\hline & AA4a & 10 & 31 & 4 & 5 & 1 & 10 & 37 & 52 & 48 \\
\hline & $\mathrm{AA} 4 \mathrm{~b}$ & 6 & 26 & 3 & 1 & 2 & 18 & 43 & 39 & 61 \\
\hline & AA5a & 11 & 25 & 6 & 7 & 0 & 11 & 41 & 48 & 52 \\
\hline & AA $5 \mathrm{~b}$ & 7 & 10 & 10 & 7 & 0 & 11 & 54 & 35 & 65 \\
\hline & $\mathrm{AA} 5 \mathrm{c}$ & 11 & 9 & 2 & 4 & 0 & 20 & 54 & 26 & 74 \\
\hline \multirow[t]{10}{*}{ Dark sand (DS) } & $\mathrm{AAGa}$ & 13 & 24 & 2 & 8 & 2 & 16 & 35 & 49 & 51 \\
\hline & $\mathrm{AACa}$ & 25 & 43 & 0 & 16 & 5 & 2 & 9 & 89 & 11 \\
\hline & $\mathrm{AA} 2 \mathrm{a}$ & 17 & 48 & 1 & 6 & 3 & 2 & 23 & 75 & 25 \\
\hline & $\mathrm{AA} 2 \mathrm{c}$ & 15 & 33 & 5 & 15 & 3 & 8 & 21 & 70 & 30 \\
\hline & AAMa & 15 & 44 & 1 & 6 & 0 & 10 & 24 & 66 & 34 \\
\hline & $\mathrm{AAMb}$ & 23 & 67 & 0 & 3 & 2 & 1 & 3 & 96 & 4 \\
\hline & AA3a & 11 & 43 & 0 & 8 & 3 & 8 & 28 & 64 & 36 \\
\hline & AA3b & 18 & 40 & 1 & 6 & 0 & 9 & 26 & 65 & 35 \\
\hline & $\mathrm{AA} 3 \mathrm{c}$ & 13 & 44 & 6 & 9 & 0 & 6 & 22 & 72 & 28 \\
\hline & $\mathrm{AAGb}$ & 39 & 40 & 0 & 15 & 1 & 0 & 5 & 95 & 5 \\
\hline \multicolumn{2}{|c|}{ Average \%HM LS } & 7.8 & 19.8 & 4.7 & 5.1 & 2.0 & 12.7 & 48.0 & 39.3 & 60.7 \\
\hline \multicolumn{2}{|l|}{ Average \%HM DS } & 18.9 & 42.6 & 1.6 & 9.2 & 1.8 & 6.3 & 19.6 & 74.2 & 25.8 \\
\hline \multicolumn{11}{|c|}{ Plage-Militaire beach (PM) } \\
\hline \multirow[t]{17}{*}{ Dark sand (DS) } & Pms6a & 9 & 25 & 5 & 15 & 5 & 6 & 34 & 60 & 40 \\
\hline & Pmsla & 28 & 38 & 3 & 19 & 4 & 3 & 5 & 92 & 8 \\
\hline & Pms2a & 32 & 46 & 1 & 11 & 2 & 1 & 8 & 91 & 9 \\
\hline & Pms3a & 38 & 50 & 2 & 2 & 1 & 1 & 5 & 94 & 6 \\
\hline & Pms4a & 39 & 45 & 2 & 6 & 1 & 2 & 4 & 94 & 6 \\
\hline & Pms5a & 36 & 41 & 2 & 16 & 0 & 2 & 3 & 95 & 5 \\
\hline & Pms1b & 9 & 44 & 1 & 24 & 4 & 1 & 15 & 83 & 17 \\
\hline & Pms2b & 37 & 41 & 2 & 15 & 1 & 0 & 3 & 96 & 4 \\
\hline & Pms3b & 29 & 50 & 1 & 15 & 1 & 2 & 3 & 95 & 5 \\
\hline & Pms4b & 14 & 53 & 2 & 16 & 1 & 1 & 13 & 86 & 14 \\
\hline & Pms5b & 35 & 48 & 0 & 10 & 0 & 4 & 4 & 92 & 8 \\
\hline & Pms6b & 19 & 46 & 0 & 21 & 2 & 1 & 12 & 88 & 13 \\
\hline & Pms1C & 32 & 55 & 1 & 7 & 1 & 0 & 3 & 97 & 3 \\
\hline & Pms2c & 35 & 41 & 1 & 17 & 0 & 1 & 5 & 94 & 6 \\
\hline & Pms3c & 33 & 52 & 4 & 6 & 0 & 1 & 3 & 95 & 5 \\
\hline & Pms5c & 29 & 52 & 2 & 11 & 0 & 1 & 5 & 93 & 7 \\
\hline & Pms6c & 46 & 39 & 1 & 10 & 2 & 0 & 2 & 98 & 2 \\
\hline \multicolumn{2}{|c|}{ Average \%HM DS } & 29.4 & 45.0 & 1.8 & 13.1 & 1.5 & 1.6 & 7.6 & 90.8 & 9.2 \\
\hline \multicolumn{11}{|c|}{ El Nasr beach (NS) } \\
\hline \multirow[t]{9}{*}{ Dark sand (DS) } & EN S 1a & 19 & 66 & 0 & 8 & 0 & 1 & 6 & 92 & 8 \\
\hline & EN S 2a & 6 & 32 & 19 & 0 & 0 & 21 & 21 & 57 & 43 \\
\hline & EN S 3a & 4 & 58 & 2 & 0 & 0 & 7 & 29 & 64 & 36 \\
\hline & EN S 1b & 9 & 66 & 7 & 7 & 0 & 3 & 9 & 88 & 12 \\
\hline & EN S $2 b$ & 18 & 72 & 5 & 0 & 0 & 0 & 5 & 95 & 5 \\
\hline & EN S 3b & 16 & 63 & 2 & 7 & 0 & 2 & 9 & 89 & 11 \\
\hline & EN S 1c & 15 & 63 & 0 & 7 & 0 & 2 & 13 & 85 & 15 \\
\hline & EN S 2c & 24 & 61 & 7 & 2 & 0 & 2 & 5 & 93 & 7 \\
\hline & EN S 3c & 17 & 59 & 4 & 10 & 0 & 3 & 7 & 90 & 10 \\
\hline Average \%HM & & 14.4 & 59.9 & 5.1 & 4.5 & 0.0 & 4.5 & 11.6 & 83.8 & 16.2 \\
\hline
\end{tabular}

The values are given as percentages of the number of grains recorded. 
(a)

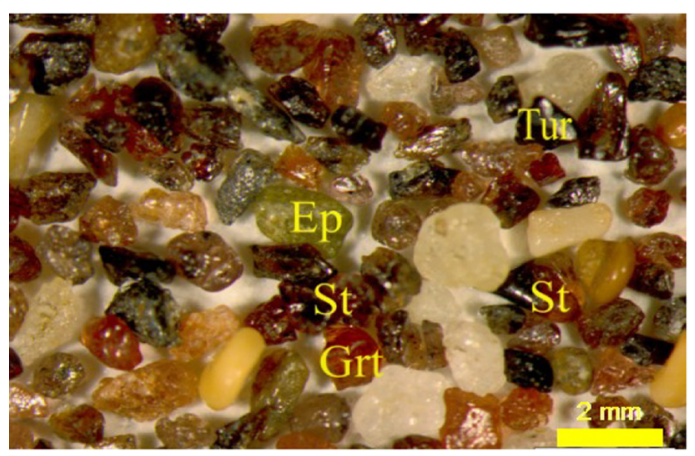

(c)

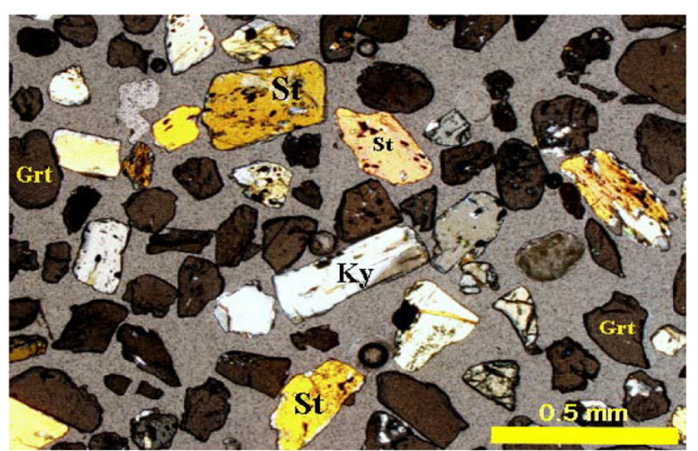

(e)

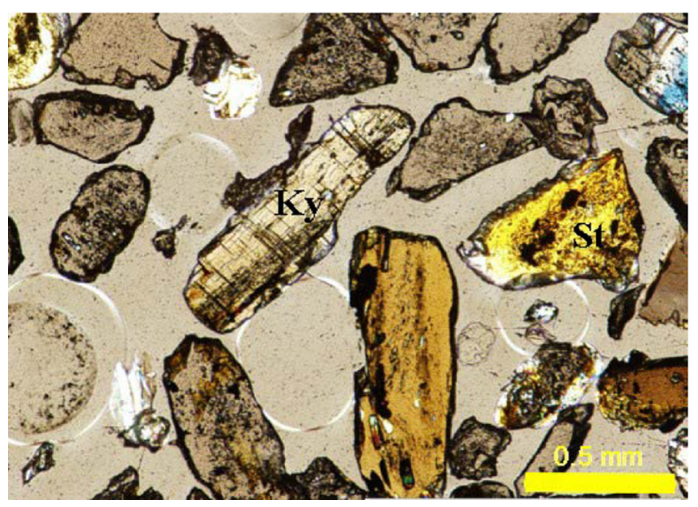

(b)

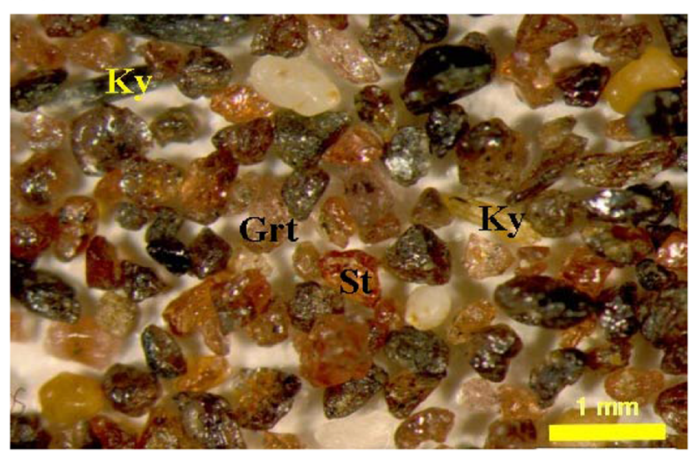

(d)

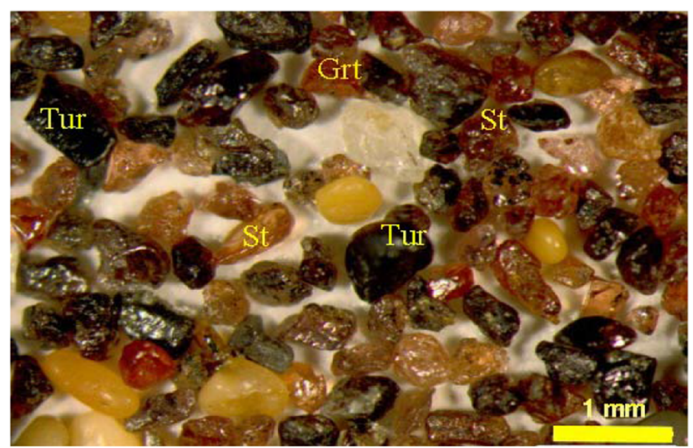

(f)

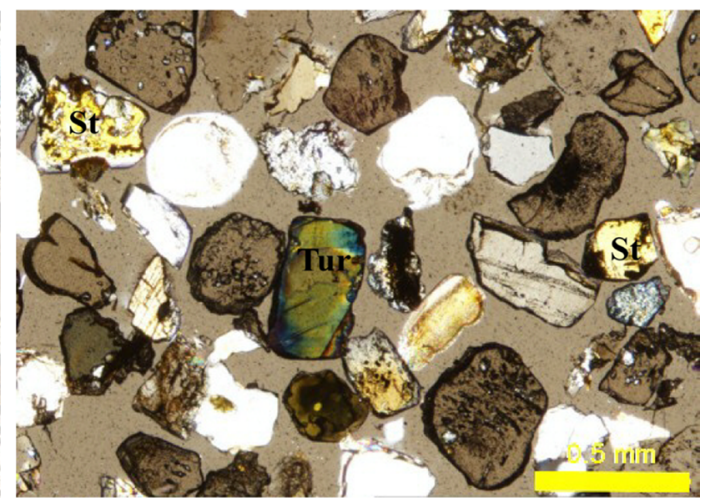

Figure 5. Microphotograph of heavy-mineral assemblage: (a, b and $\mathbf{c})$ from Ain Achir and Plage-Militaire beaches; (d, e, and f) from El Nasr beach.

prismatic fragments with typical step-like fractures (figure 5e) and rounded and elongated tourmaline grains (figure 5d and $\mathrm{f}$ ). Epidote is not present in the El Nasr sand samples.

\subsubsection{Heavy-mineral inclusions}

Most of the heavy minerals of the three beaches have solid inclusions. Hand-picked single garnet, staurolite and kyanite grains with solid inclusions from the beaches have been analysed by X-ray diffraction (XRD). The detrital garnets from the Ain Achir, Plage-Militaire and El Nasr sands show different types of inclusions such as quartz (figure 6a). At the Ain Achir, most of the staurolite grains have garnet inclusions (figure 6b) and those from the Plage-Militaire and El Nasr sands have tourmaline, garnet and zircon inclusions (figure 6c). Kyanite grains from the three beaches show no differences. Their inclusions are mostly garnet or quartz (figure 6d).

\subsection{Sand mineral chemistry}

\subsubsection{Garnet}

The structural formula of the analysed garnets was calculated according to Locock (2008) on the 
Table 2. Chemical composition and end-member compositions of the garnets from the three beaches.

\begin{tabular}{|c|c|c|c|c|c|c|c|c|c|c|c|c|c|c|}
\hline & $\mathrm{SiO}_{2}$ & $\mathrm{TiO}_{2}$ & $\mathrm{Al}_{2} \mathrm{O}_{3}$ & $\mathrm{FeO}$ & $\mathrm{MnO}$ & $\mathrm{MgO}$ & $\mathrm{CaO}$ & Total & Alm & Sps & Prp & Grs & Adr & Uv \\
\hline \multicolumn{15}{|c|}{ Ain Achir beach } \\
\hline AAS1 & 37.0 & 0.1 & 21.4 & 38.7 & 0.2 & 2.0 & 0.5 & 100.0 & 90 & 0 & 8 & 2 & 0 & 0 \\
\hline AAS2 & 36.9 & 0.1 & 21.3 & 39.0 & 0.1 & 1.9 & 0.5 & 99.9 & 90 & 0 & 8 & 2 & 0 & 0 \\
\hline AAS3 & 36.9 & 0.1 & 21.3 & 39.0 & 0.2 & 2.0 & 0.6 & 100.0 & 90 & 0 & 8 & 2 & 0 & 0 \\
\hline AAS4 & 37.0 & 0.1 & 21.3 & 39.0 & 0.1 & 2.0 & 0.6 & 100.1 & 90 & 0 & 8 & 2 & 0 & 0 \\
\hline AAS5 & 37.8 & 0.1 & 21.4 & 35.0 & 1.2 & 2.4 & 2.1 & 99.9 & 82 & 3 & 10 & 6 & 0 & 0 \\
\hline AAS6 & 37.7 & 0.1 & 21.3 & 35.1 & 1.2 & 2.4 & 2.3 & 100.0 & 81 & 3 & 9 & 7 & 0 & 0 \\
\hline AAS7 & 37.7 & 0.1 & 21.4 & 34.9 & 1.2 & 2.3 & 2.2 & 99.9 & 82 & 3 & 9 & 7 & 0 & 0 \\
\hline AAS8 & 37.6 & 0.1 & 21.4 & 35.1 & 1.3 & 2.2 & 2.2 & 99.9 & 82 & 3 & 9 & 7 & 0 & 0 \\
\hline AAS9 & 37.6 & 0.1 & 21.5 & 34.9 & 1.3 & 2.2 & 2.1 & 99.8 & 82 & 3 & 9 & 6 & 0 & 0 \\
\hline AAS10 & 37.9 & 0.1 & 21.1 & 36.5 & 0.2 & 3.2 & 1.0 & 100.0 & 84 & 0 & 12 & 3 & 0 & 0 \\
\hline AAS11 & 37.7 & 0.0 & 21.3 & 36.7 & 0.2 & 3.1 & 1.0 & 99.9 & 85 & 0 & 12 & 3 & 0 & 0 \\
\hline AAS12 & 37.7 & 0.1 & 21.3 & 36.5 & 0.2 & 2.9 & 1.2 & 99.9 & 85 & 0 & 12 & 3 & 0 & 0 \\
\hline AAS13 & 37.7 & 0.1 & 21.2 & 36.7 & 0.2 & 2.9 & 1.2 & 99.9 & 85 & 0 & 11 & 4 & 0 & 0 \\
\hline AAS14 & 37.7 & 0.1 & 21.2 & 36.6 & 0.1 & 2.9 & 1.2 & 99.8 & 85 & 0 & 11 & 3 & 0 & 0 \\
\hline AAS15 & 37.7 & 0.1 & 21.2 & 36.8 & 0.2 & 2.7 & 1.1 & 99.9 & 86 & 0 & 11 & 3 & 0 & 0 \\
\hline AAS16 & 37.5 & 0.1 & 21.2 & 37.3 & 0.2 & 2.6 & 1.1 & 100.1 & 86 & 0 & 10 & 3 & 0 & 0 \\
\hline AAS17 & 37.8 & 0.1 & 21.2 & 37.2 & 0.2 & 2.5 & 1.1 & 100.0 & 87 & 0 & 10 & 3 & 0 & 0 \\
\hline AAS18 & 37.6 & 0.1 & 21.1 & 35.4 & 1.3 & 1.1 & 3.5 & 100.1 & 83 & 3 & 4 & 10 & 0 & 0 \\
\hline AAS19 & 37.4 & 0.1 & 21.1 & 34.7 & 1.3 & 1.1 & 4.1 & 99.9 & 81 & 3 & 4 & 12 & 0 & 0 \\
\hline AAS20 & 37.4 & 0.1 & 21.3 & 34.3 & 1.5 & 1.0 & 4.6 & 100.1 & 80 & 3 & 4 & 13 & 0 & 0 \\
\hline
\end{tabular}

\section{Plage-Militaire beach}

\begin{tabular}{|c|c|c|c|c|c|c|c|c|c|c|c|c|c|}
\hline PMS1 & 37.6 & 0.0 & 22.5 & 34.3 & 0.0 & 5.1 & 0.5 & 100.1 & 78 & 0 & 20 & 2 & 0 \\
\hline PMS2 & 37.3 & 0.0 & 22.1 & 36.1 & 0.0 & 4.0 & 0.4 & 100.0 & 83 & 0 & 16 & 1 & 0 \\
\hline PMS3 & 37.3 & 0.0 & 21.9 & 36.7 & 0.0 & 3.7 & 0.4 & 100.0 & 84 & 0 & 15 & 1 & 0 \\
\hline PMS4 & 37.1 & 0.1 & 21.9 & 36.8 & 0.1 & 3.7 & 0.4 & 100.0 & 84 & 0 & 15 & 1 & 0 \\
\hline PMS5 & 37.7 & 0.0 & 22.1 & 36.2 & 0.0 & 3.7 & 0.4 & 100.0 & 84 & 0 & 15 & 1 & 0 \\
\hline PMS6 & 37.5 & 0.0 & 22.0 & 36.4 & 0.0 & 3.9 & 0.4 & 100.1 & 84 & 0 & 15 & 1 & 0 \\
\hline PMS7 & 37.5 & 0.0 & 22.2 & 35.4 & 0.0 & 4.5 & 0.4 & 100.0 & 81 & 0 & 18 & 1 & 0 \\
\hline PMS8 & 37.8 & 0.1 & 22.0 & 35.0 & 0.0 & 4.5 & 0.5 & 99.9 & 81 & 0 & 18 & 1 & 0 \\
\hline PMS9 & 36.7 & 0.1 & 21.7 & 36.3 & 2.1 & 1.6 & 1.3 & 99.9 & 85 & 5 & 7 & 4 & 0 \\
\hline PMS10 & 36.5 & 0.1 & 21.7 & 36.6 & 1.6 & 2.1 & 1.4 & 100.0 & 84 & 4 & 9 & 4 & 0 \\
\hline PMS11 & 36.6 & 0.1 & 21.7 & 36.1 & 1.6 & 2.2 & 1.6 & 100.0 & 83 & 4 & 9 & 5 & 0 \\
\hline PMS12 & 36.6 & 0.1 & 21.8 & 35.9 & 1.7 & 2.1 & 1.6 & 99.9 & 83 & 4 & 8 & 5 & 0 \\
\hline PMS13 & 36.9 & 0.1 & 21.7 & 35.8 & 1.7 & 2.0 & 1.7 & 99.9 & 83 & 4 & 8 & 5 & 0 \\
\hline PMS14 & 37.1 & 0.1 & 21.8 & 35.2 & 1.7 & 2.0 & 2.1 & 100.1 & 82 & 4 & 8 & 6 & 0 \\
\hline PMS15 & 37.0 & 0.1 & 21.8 & 35.7 & 1.7 & 2.0 & 1.8 & 100.1 & 83 & 4 & 8 & 5 & 0 \\
\hline PMS16 & 36.6 & 0.2 & 21.8 & 36.4 & 1.6 & 2.0 & 1.4 & 100.0 & 84 & 4 & 8 & 4 & 0 \\
\hline PMS17 & 36.4 & 0.2 & 22.0 & 36.3 & 2.0 & 1.8 & 1.3 & 99.9 & 84 & 5 & 7 & 4 & 0 \\
\hline PMS18 & 37.5 & 0.1 & 21.3 & 28.1 & 2.7 & 1.2 & 8.7 & 99.7 & 64 & 6 & 5 & 25 & 0 \\
\hline PMS19 & 37.4 & 0.1 & 21.3 & 28.2 & 3.1 & 1.0 & 8.8 & 99.9 & 64 & 7 & 4 & 25 & 0 \\
\hline PMS20 & 37.5 & 0.1 & 21.2 & 28.4 & 3.1 & 1.0 & 8.8 & 100.1 & 64 & 7 & 4 & 25 & 0 \\
\hline PMS21 & 37.5 & 0.1 & 21.4 & 28.6 & 3.2 & 0.9 & 8.4 & 100.0 & 65 & 7 & 4 & 24 & 0 \\
\hline PMS22 & 37.2 & 0.1 & 21.3 & 29.0 & 3.1 & 0.9 & 8.4 & 100.1 & 65 & 7 & 4 & 24 & 0 \\
\hline PMS23 & 37.3 & 0.1 & 21.3 & 29.3 & 2.8 & 1.0 & 8.0 & 99.9 & 67 & 6 & 4 & 23 & 0 \\
\hline PMS24 & 37.7 & 0.1 & 21.4 & 29.4 & 2.3 & 1.1 & 8.1 & 100.0 & 68 & 5 & 4 & 24 & 0 \\
\hline PMS25 & 36.6 & 0.0 & 21.5 & 34.8 & 0.5 & 0.8 & 5.5 & 99.8 & 80 & 1 & 3 & 16 & 0 \\
\hline PMS26 & 36.5 & 0.1 & 21.4 & 35.0 & 0.5 & 0.7 & 5.9 & 100.1 & 79 & 1 & 3 & 17 & 0 \\
\hline PMS27 & 36.7 & 0.1 & 21.5 & 34.6 & 0.5 & 0.7 & 5.8 & 100.0 & 79 & 1 & 3 & 17 & 0 \\
\hline PMS28 & 36.7 & 0.1 & 21.5 & 35.1 & 0.3 & 0.7 & 5.4 & 100.0 & 80 & 1 & 3 & 16 & 0 \\
\hline PMS29 & 37.0 & 0.1 & 21.5 & 34.8 & 0.4 & 0.7 & 5.6 & 100.1 & 80 & 1 & 3 & 16 & 0 \\
\hline PMS30 & 36.9 & 0.1 & 21.3 & 35.5 & 0.3 & 0.8 & 5.0 & 100.0 & 81 & 1 & 3 & 15 & 0 \\
\hline PMS31 & 36.7 & 0.1 & 21.3 & 35.7 & 0.3 & 0.8 & 4.9 & 99.9 & 82 & 1 & 3 & 14 & 0 \\
\hline
\end{tabular}


Table 2. (Continued.)

\begin{tabular}{|c|c|c|c|c|c|c|c|c|c|c|c|c|c|c|}
\hline & $\mathrm{SiO}_{2}$ & $\mathrm{TiO}_{2}$ & $\mathrm{Al}_{2} \mathrm{O}_{3}$ & $\mathrm{FeO}$ & $\mathrm{MnO}$ & $\mathrm{MgO}$ & $\mathrm{CaO}$ & Total & Alm & Sps & Prp & Grs & Adr & Uv \\
\hline PMS32 & 37.0 & 0.1 & 21.3 & 34.5 & 0.6 & 1.2 & 5.1 & 99.9 & 79 & 1 & 5 & 15 & 0 & 0 \\
\hline PMS33 & 37.1 & 0.1 & 21.4 & 34.3 & 0.6 & 1.2 & 5.2 & 100.0 & 79 & 1 & 5 & 15 & 0 & 0 \\
\hline PMS34 & 37.0 & 0.1 & 21.7 & 34.0 & 0.6 & 1.2 & 5.2 & 99.9 & 79 & 1 & 5 & 15 & 0 & 0 \\
\hline PMS35 & 37.0 & 0.1 & 21.5 & 34.6 & 0.5 & 1.3 & 5.0 & 100.0 & 79 & 1 & 5 & 15 & 0 & 0 \\
\hline PMS36 & 37.3 & 0.1 & 21.2 & 34.3 & 0.6 & 1.2 & 5.3 & 100.0 & 79 & 1 & 5 & 16 & 0 & 0 \\
\hline PMS37 & 36.8 & 0.1 & 21.5 & 35.8 & 0.5 & 1.3 & 4.2 & 100.1 & 82 & 1 & 5 & 12 & 0 & 0 \\
\hline PMS38 & 37.1 & 0.1 & 21.4 & 35.4 & 0.3 & 1.5 & 4.1 & 100.0 & 82 & 1 & 6 & 12 & 0 & 0 \\
\hline \multicolumn{15}{|c|}{ El Nasr beach } \\
\hline EN1 & 37.7 & 0.0 & 21.7 & 34.9 & 0.2 & 4.8 & 0.8 & 100.1 & 79 & 0 & 19 & 2 & 0 & 0 \\
\hline EN2 & 37.8 & 0.0 & 21.6 & 33.8 & 0.5 & 3.8 & 2.4 & 99.9 & 77 & 1 & 15 & 7 & 0 & 0 \\
\hline EN3 & 38.2 & 0.1 & 21.9 & 33.1 & 0.3 & 4.3 & 2.3 & 100.1 & 75 & 1 & 18 & 6 & 0 & 0 \\
\hline EN4 & 38.3 & 0.0 & 21.5 & 33.1 & 0.7 & 4.0 & 2.5 & 100.1 & 76 & 2 & 16 & 7 & 0 & 0 \\
\hline
\end{tabular}

Alm: Almandine, Sps: Spessartine, Prp: Pyrope, Adr: Andradite, Uv: Uvarovite, Grs: Grossular (Whitney and Evans 2010).

basis of 12 oxygen atoms (table 2). Although all analysed garnet grains represent a chemical mixture of the different components, they are extremely rich in almandine component. Their chemical compositions are plotted in the Alm-Prp-Grs diagram (figure 7).

Garnets at the Ain Achir beach have a relatively homogeneous end-member composition: (Prp 4$12 \mathrm{~mol} \%$, Alm 81-90 mol\%, Grs 2-13 mol\%) (figure 7a). The Plage-Militaire sand is characterised by four kinds of garnet composition (figure 7b): (i) (Prp 15-20 mol\%, Alm 78-84 mol\%, Grs 1-2 mol\%), (ii) (Prp 7-9 mol\%, Alm 82$85 \mathrm{~mol} \%$, Grs 4-6 mol\%), (iii) (Prp 4-5 mol\%, Alm 64-68 mol\%, Grs 23-25 mol\%) and (iv) (Prp 3-6 mol\%, Alm 79-82 mol\%, Grs 12-17 mol\%). In contrast to those from the PlageMilitaire, the compositions of garnet of El Nasr sands show a higher pyrope end-member content (Prp 16-19 mol\%, Alm 75-79 mol\%, Grs 2-7 mol\%) (figure $7 \mathrm{c}$ ).

\subsubsection{Tourmaline}

The program WinTcac (Yavuz et al. 2006) has been used for mineral recalculation and classification of tourmaline. The normalisation approach based on the $\mathrm{Si}=15$ (apfu) is useful for tourmaline analyses with unanalysed elements such as $\mathrm{Li}$ and $\mathrm{B}$ and is appropriate for tourmalines from metamorphic rocks (Henry et al. 2011). The composition of tourmalines from each beach type is presented in table 3 .
All tourmaline samples plot in the alkalitourmaline group field on the $\mathrm{Ca}-\mathrm{X}_{\mathrm{vac}}-(\mathrm{Na}+\mathrm{K})$ ternary diagram (figure 8a-c) of Jiang et al. (1995, 1996). Detrital tourmaline of the Ain Achir and Plage-Militaire beaches is $\mathrm{Fe}$ rich with $\mathrm{X}_{\mathrm{Fe}}=$ $(\mathrm{Fe} / \mathrm{Fe}+\mathrm{Mg})$ varying between 0.76 and 0.94 , respectively; therefore, they are schorlitic in their composition (figure $8 \mathrm{~d}$ and e). Tourmalines from the El Nasr beach are Fe-poor with a mean value of $\mathrm{X}_{\mathrm{Fe}}=0.26$ and plot in the dravite field (figure $8 \mathrm{f}$ ).

\subsection{Geochemistry of whole sand and basement}

At the Ain Achir beach, the light and dark sands show some differences in their chemical compositions (table 4a). The average total REE content at the Ain Achir beach is 69 and 128 ppm for the light and dark sands, respectively. The Ain Achir light sands are mainly high in LREE $(\Sigma L R E E=56 \mathrm{ppm})$ rather than HREE $(\Sigma$ HREE $=12 \mathrm{ppm})$. Whereas the dark sand shows higher LREE and HREE concentrations $(\Sigma \mathrm{LREE}=100 \mathrm{ppm}$ and $\Sigma \mathrm{HREE}=28 \mathrm{ppm})$. Compared to the REE data from the Ain Achir, the Plage-Militaire sands show somewhat higher values of $\Sigma$ REE, $\Sigma$ LREE and $\Sigma$ HREE: $\Sigma$ REE $=$ 130-274 ppm, average $230 \mathrm{ppm} ; \Sigma \mathrm{LREE}=141-$ $198 \mathrm{ppm}$, average $168 \mathrm{ppm}$ and $\mathrm{HHREE}=$ 41-75 ppm, average $61 \mathrm{ppm}$ (table 4a). At the El Nasr (table 4a), the averages are: $\Sigma R E E$ $=196 \mathrm{ppm}, \Sigma \mathrm{LREE}=149 \mathrm{ppm}$ and $\Sigma \mathrm{HREE}=$ 47 ppm. 

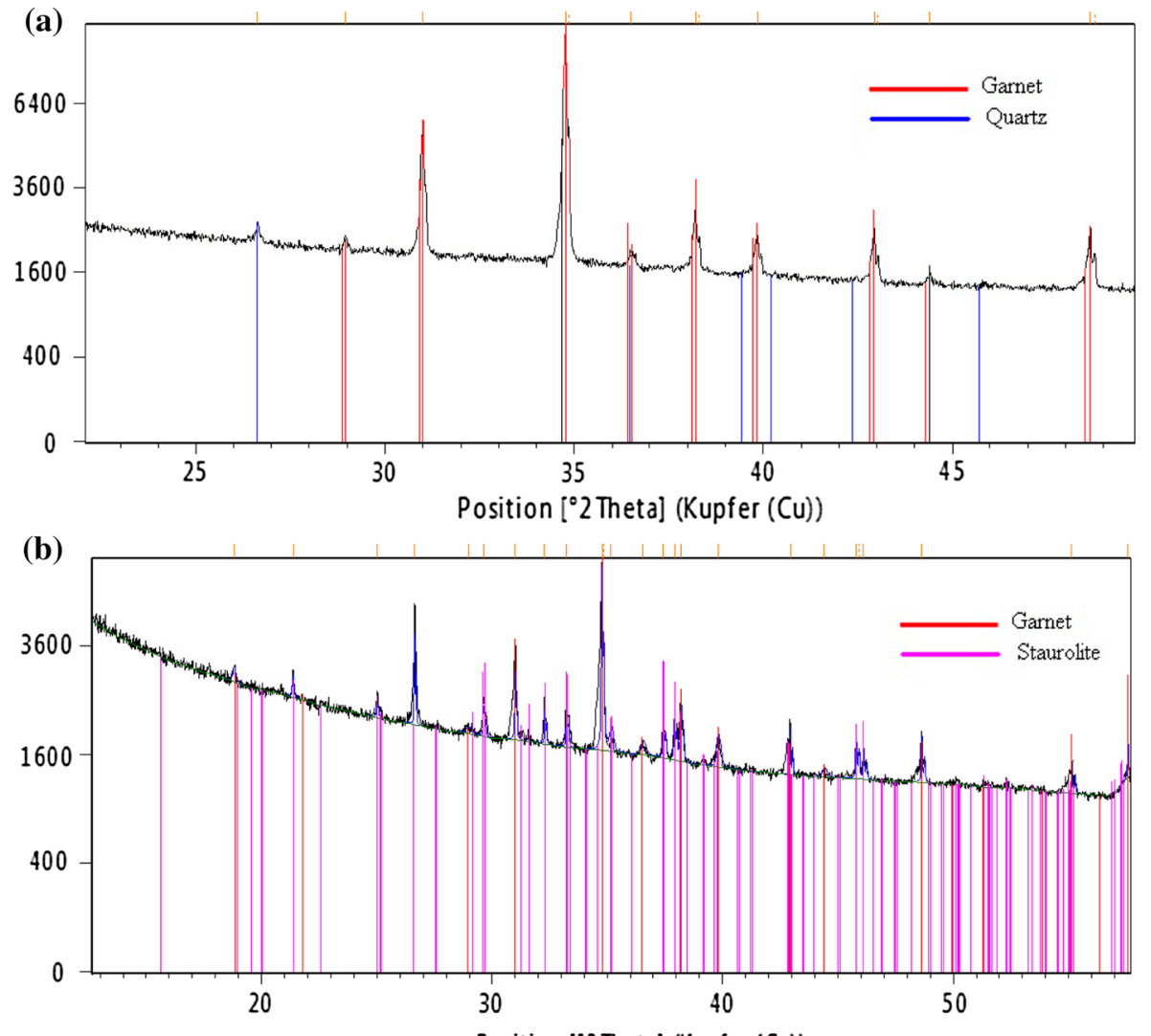

(c)

Position [ 2 Theta] (Kupfer (Cu))
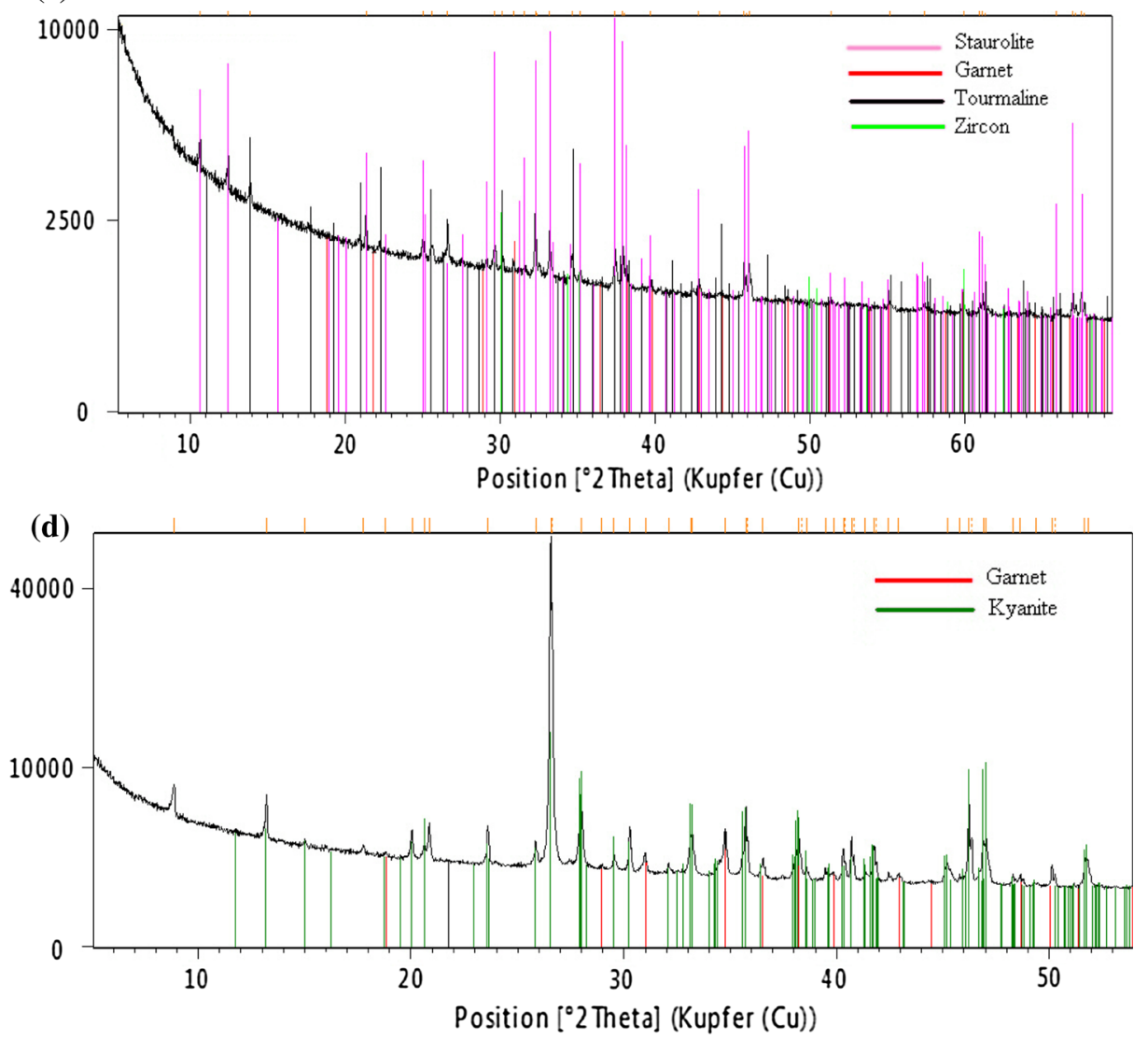

Figure 6. XRD diagrams of (a) detrital garnets with quartz inclusions, (b) staurolite grains with garnet inclusions, (c) staurolite grains with tourmaline, zircon and garnet inclusions, and (d) kyanite grains with garnet inclusion. 

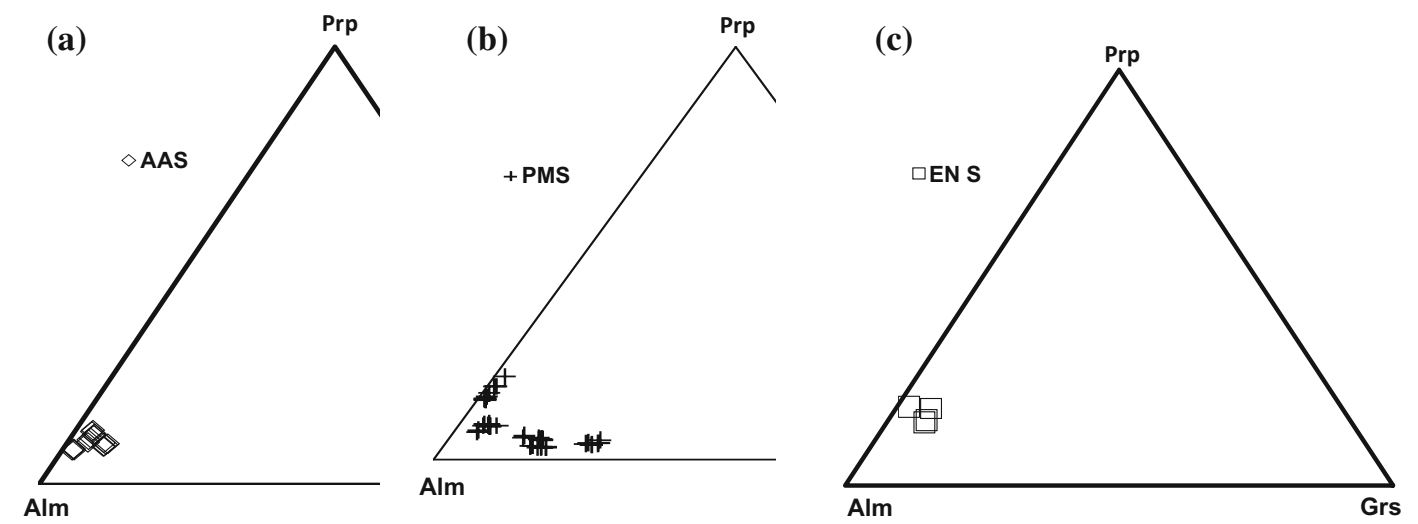

Figure 7. Ternary garnet composition diagrams showing abundances of almandine, grossular and pyrope end-members from the heavy-mineral sand beaches. AA S: Ain Achir sand, PM S: Plage-Militaire sand, EN S: El Nasr sand.

The average REE values of the kyanite-bearing micaschists from the Vivier zone at the Cap de Garde (R Av. REE VIV) are clearly higher: $\Sigma$ REE $=249 \mathrm{ppm}, \quad \Sigma \mathrm{LREE}=218 \mathrm{ppm}$ and $\Sigma$ HREE $=30 \mathrm{ppm}$ than those of the Ain Achir garnetstaurolite micaschist outcrops (R Av. REE AA): $\Sigma \mathrm{REE}=207 \mathrm{ppm}, \quad \Sigma \mathrm{LREE}=182 \mathrm{ppm}$ and $\Sigma$ HREE $=24 \mathrm{ppm}$ (table $4 \mathrm{~b}$ ). At the Plage-Militaire, the REE concentrations are nearly similar and their $\Sigma$ REE $=148 \mathrm{ppm}, \mathrm{\Sigma LREE}=129 \mathrm{ppm}$ and $\Sigma$ HREE $=18 \mathrm{ppm}$. The El Nasr rock outcrops have high average REE values: $\Sigma$ REE $=218 \mathrm{ppm}$, $\Sigma \mathrm{LREE}=193 \mathrm{ppm}$ and $\mathrm{SHREE}=25 \mathrm{ppm}$ (table $4 b$ ).

\section{Discussion of the provenance}

Heavy-mineral (specific gravity 2.85 or greater) assemblages reflect the mineralogy of the source rocks. Mineral chemistry of heavy minerals such as garnet (Mange and Wright 2007) and tourmaline (Henry and Guidotti 1985) is applicable to provenance studies and they are useful indicators of the nature of the source area.

\subsection{Garnet}

Garnet is resistant to weathering, and has compositions that may reflect the composition of the source rocks. Garnet shows significant differences in the composition between different types of garnet-bearing lithologies. In general, detrital garnet is considered to mostly originate from metamorphic rocks (Blatt et al. 2006).

The Ain Achir sand garnet (Prp-Alm-Grs) compositions are relatively homogeneous. In figure $9(\mathrm{a})$, all samples are grouped in the field $\mathrm{Bi}$ for intermediate to felsic igneous rocks suggesting a single-source type. The single source of garnet at Ain Achir can be explained by the presence of only one variety of garnet (almandine rich) in the micaschist outcrop (e.g., garnet-staurolite micaschist). The garnets from the Plage-Militaire sand split into two groups with a noticeable difference in garnet end-member composition (figure 9b). The first group is dominated by almandine-pyrope with low grossular content and the second by higher grossular and lower pyrope concentration. The presence of two garnet compositions might result from mixed sources (probably the proximal garnetstaurolite micaschists and the garnet micaschist) or multiple garnet varieties in a single source). The garnet samples plot both in the field $\mathrm{Bi}$ for intermediate to felsic igneous rocks and in the field B for amphibolite-facies metasedimentary rocks. The difference in abundance of garnet varieties in the Plage-Militaire garnet sand samples may also reflect mixed sources (e.g., proximal garnetstaurolite micaschists and garnet micaschists). The El Nasr garnets are homogeneous and show a relatively high pyrope component. All samples plot in the field $\mathrm{Bi}$ for intermediate to felsic igneous rocks. Their high abundance in pyrope reflects a single magnesian-rich source (figure 9c).

\subsection{Tourmaline}

The source-rock types of detrital tourmaline can be identified with the triangular $\mathrm{Ca}-\mathrm{Fe}-\mathrm{Mg}$ diagrams of Henry and Guidotti (1985). Comparison of the tourmaline data from the studied sands with compositional fields of tourmaline of known origins (figure 10) indicates a strong diversity of the source rock. According to the $\mathrm{Fe} /(\mathrm{Fe}+\mathrm{Mg})$, $\mathrm{Mg} /(\mathrm{Mg}+\mathrm{Fe})$ ratio and $\mathrm{Ca}^{2+}$ content, tourmaline 
Table 3. Composition of tourmalines from each beach.

\begin{tabular}{|c|c|c|c|c|c|c|c|c|c|c|c|}
\hline & $\mathrm{SiO}_{2}$ & $\mathrm{TiO}_{2}$ & $\mathrm{Al}_{2} \mathrm{O}_{3}$ & $\mathrm{FeO}$ & $\mathrm{MnO}$ & $\mathrm{MgO}$ & $\mathrm{CaO}$ & $\mathrm{Na}_{2} \mathrm{O}$ & $\mathrm{K}_{2} \mathrm{O}$ & $\mathrm{B}_{2} \mathrm{O}_{3}$ & Total \\
\hline \multicolumn{12}{|c|}{ Ain Achir beach } \\
\hline AAS1 & 34.93 & 0.44 & 36.30 & 11.54 & 0.07 & 2.50 & 0.21 & 1.89 & 0.03 & 9.99 & 97.89 \\
\hline AAS2 & 35.14 & 0.46 & 36.41 & 11.76 & 0.10 & 2.53 & 0.23 & 1.87 & 0.04 & 10.05 & 98.58 \\
\hline AAS3 & 35.06 & 0.50 & 35.99 & 11.75 & 0.09 & 2.58 & 0.24 & 1.87 & 0.03 & 10.03 & 98.13 \\
\hline AAS4 & 35.01 & 0.51 & 36.01 & 11.68 & 0.08 & 2.61 & 0.23 & 1.95 & 0.03 & 10.02 & 98.13 \\
\hline AAS5 & 34.99 & 0.51 & 35.87 & 11.77 & 0.09 & 2.64 & 0.21 & 1.98 & 0.04 & 10.01 & 98.12 \\
\hline AAS6 & 34.84 & 0.49 & 35.90 & 11.88 & 0.08 & 2.59 & 0.23 & 1.92 & 0.04 & 9.97 & 97.95 \\
\hline AAS7 & 35.15 & 0.50 & 35.73 & 11.66 & 0.09 & 2.67 & 0.22 & 1.96 & 0.04 & 10.06 & 98.10 \\
\hline AAS8 & 35.15 & 0.48 & 35.63 & 11.88 & 0.04 & 2.69 & 0.23 & 1.93 & 0.04 & 10.06 & 98.15 \\
\hline AAS9 & 35.00 & 0.51 & 35.80 & 11.79 & 0.08 & 2.64 & 0.22 & 2.02 & 0.04 & 10.01 & 98.13 \\
\hline AAS10 & 34.83 & 0.55 & 36.14 & 11.90 & 0.06 & 2.60 & 0.23 & 2.20 & 0.04 & 9.97 & 98.53 \\
\hline AAS11 & 34.46 & 0.52 & 36.15 & 11.66 & 0.04 & 2.49 & 0.25 & 2.31 & 0.04 & 9.86 & 97.78 \\
\hline AAS12 & 34.62 & 0.45 & 36.31 & 11.68 & 0.09 & 2.46 & 0.27 & 1.90 & 0.04 & 9.90 & 97.72 \\
\hline AAS13 & 34.64 & 0.49 & 36.17 & 11.81 & 0.04 & 2.52 & 0.25 & 2.36 & 0.05 & 9.91 & 98.25 \\
\hline AAS14 & 34.85 & 0.50 & 36.18 & 11.73 & 0.07 & 2.52 & 0.25 & 2.25 & 0.05 & 9.97 & 98.36 \\
\hline AAS15 & 34.80 & 0.50 & 36.26 & 11.76 & 0.07 & 2.53 & 0.24 & 2.40 & 0.04 & 9.96 & 98.56 \\
\hline AAS16 & 34.62 & 0.53 & 36.11 & 11.66 & 0.06 & 2.56 & 0.25 & 2.44 & 0.05 & 9.91 & 98.18 \\
\hline AAS17 & 34.54 & 0.45 & 36.15 & 11.96 & 0.08 & 2.57 & 0.25 & 2.33 & 0.04 & 9.88 & 98.28 \\
\hline AAS18 & 34.62 & 0.49 & 36.23 & 11.99 & 0.05 & 2.36 & 0.25 & 2.32 & 0.04 & 9.91 & 98.28 \\
\hline AAS19 & 34.84 & 0.48 & 36.24 & 12.05 & 0.10 & 2.45 & 0.26 & 2.34 & 0.05 & 9.97 & 98.79 \\
\hline AAS20 & 36.01 & 0.70 & 34.56 & 8.31 & 0.00 & 4.61 & 1.05 & 2.28 & 0.06 & 10.30 & 97.91 \\
\hline AAS21 & 36.00 & 0.74 & 34.74 & 8.44 & 0.04 & 4.60 & 1.06 & 2.38 & 0.05 & 10.30 & 98.37 \\
\hline AAS22 & 35.77 & 0.73 & 34.78 & 8.27 & 0.02 & 4.66 & 0.99 & 2.37 & 0.06 & 10.24 & 97.88 \\
\hline AAS23 & 36.00 & 0.70 & 34.95 & 8.31 & 0.03 & 4.68 & 1.03 & 2.42 & 0.06 & 10.30 & 98.52 \\
\hline AAS24 & 35.57 & 0.77 & 34.75 & 8.39 & 0.00 & 4.60 & 1.01 & 2.36 & 0.07 & 10.18 & 97.73 \\
\hline AAS25 & 35.97 & 0.71 & 34.80 & 8.34 & 0.03 & 4.61 & 0.96 & 2.10 & 0.05 & 10.30 & 97.88 \\
\hline AAS26 & 36.00 & 0.67 & 34.78 & 8.44 & 0.02 & 4.62 & 0.95 & 2.15 & 0.06 & 10.30 & 98.04 \\
\hline AAS27 & 35.70 & 0.45 & 36.20 & 10.52 & 0.09 & 2.33 & 0.38 & 1.76 & 0.03 & 10.22 & 97.68 \\
\hline AAS28 & 35.72 & 0.52 & 34.85 & 10.70 & 0.01 & 2.67 & 0.33 & 1.84 & 0.03 & 10.22 & 96.91 \\
\hline AAS29 & 36.11 & 0.44 & 35.52 & 10.50 & 0.05 & 2.68 & 0.31 & 1.82 & 0.04 & 10.33 & 97.85 \\
\hline AAS30 & 35.96 & 0.49 & 35.07 & 10.67 & 0.07 & 2.74 & 0.33 & 1.91 & 0.05 & 10.29 & 97.63 \\
\hline AAS31 & 35.87 & 0.71 & 34.50 & 11.11 & 0.03 & 2.85 & 0.36 & 1.99 & 0.05 & 10.26 & 97.74 \\
\hline AAS32 & 35.87 & 0.65 & 33.90 & 10.89 & 0.05 & 3.17 & 0.46 & 1.98 & 0.04 & 10.26 & 97.27 \\
\hline AAS33 & 35.73 & 0.51 & 34.51 & 10.99 & 0.08 & 2.94 & 0.42 & 1.93 & 0.05 & 10.22 & 97.43 \\
\hline \multicolumn{12}{|c|}{ Plage-Militaire beach } \\
\hline PMS1 & 34.00 & 1.00 & 33.00 & 9.00 & 0.00 & 4.66 & 0.38 & 2.01 & 0.05 & 10.10 & 94.19 \\
\hline PMS2 & 34.00 & 0.00 & 34.00 & 11.00 & 0.00 & 3.26 & 0.35 & 2.12 & 0.07 & 10.17 & 94.96 \\
\hline PMS3 & 33.00 & 0.00 & 34.00 & 13.00 & 0.00 & 1.63 & 0.31 & 2.08 & 0.04 & 9.90 & 93.96 \\
\hline PMS4 & 33.00 & 0.00 & 33.00 & 13.00 & 0.00 & 2.16 & 0.34 & 2.14 & 0.04 & 9.94 & 93.62 \\
\hline PMS5 & 33.00 & 0.00 & 34.00 & 13.00 & 0.00 & 1.65 & 0.33 & 2.03 & 0.06 & 9.90 & 93.96 \\
\hline PMS6 & 33.00 & 0.00 & 34.00 & 12.00 & 0.00 & 2.18 & 0.33 & 2.14 & 0.05 & 9.98 & 93.68 \\
\hline \multicolumn{12}{|c|}{ El Nasr beach } \\
\hline EN S1 & 36.00 & 0.00 & 33.00 & 5.00 & 0.00 & 7.65 & 0.89 & 2.14 & 0.02 & 10.57 & 84.57 \\
\hline EN S2 & 36.00 & 0.00 & 33.00 & 5.00 & 0.00 & 7.31 & 1.16 & 2.09 & 0.02 & 10.43 & 84.43 \\
\hline EN S3 & 36.00 & 0.00 & 32.00 & 5.00 & 0.00 & 7.66 & 0.85 & 2.07 & 0.03 & 10.35 & 83.35 \\
\hline EN S4 & 36.00 & 0.00 & 30.00 & 6.00 & 0.00 & 8.94 & 0.98 & 2.31 & 0.06 & 10.39 & 82.39 \\
\hline EN S5 & 36.00 & 0.00 & 31.00 & 5.00 & 0.00 & 9.32 & 0.72 & 2.31 & 0.01 & 10.50 & 82.50 \\
\hline EN S6 & 35.00 & 0.00 & 31.00 & 6.00 & 0.00 & 8.68 & 1.62 & 1.78 & 0.07 & 10.37 & 82.37 \\
\hline
\end{tabular}


Table 3. (Continued.)

\begin{tabular}{|c|c|c|c|c|c|c|c|c|c|c|}
\hline & $\mathrm{Fe}_{2}+(\mathrm{Y})$ & $\operatorname{Mg}(\mathrm{Y})$ & $\mathrm{Ca}(\mathrm{X})$ & $\mathrm{Na}(\mathrm{X})$ & $\mathrm{K}(\mathrm{X})$ & X-vacancy & $\mathrm{Y}(\mathrm{Fe} /(\mathrm{Fe}+\mathrm{Mg}))$ & $\mathrm{Mg} /(\mathrm{Mg}+\mathrm{Fe})$ & $\mathrm{X}(\mathrm{ca} / \mathrm{Ca}+\mathrm{Na})$ & $\mathrm{Na}+\mathrm{K}$ \\
\hline \multicolumn{11}{|c|}{ Ain Achir beach } \\
\hline AAS1 & 1.66 & 0.64 & 0.04 & 0.63 & 0.01 & 0.33 & 0.94 & 0.28 & 0.06 & 0.64 \\
\hline AAS2 & 1.68 & 0.64 & 0.04 & 0.62 & 0.01 & 0.33 & 0.94 & 0.28 & 0.06 & 0.63 \\
\hline AAS3 & 1.68 & 0.66 & 0.04 & 0.62 & 0.01 & 0.33 & 0.93 & 0.28 & 0.07 & 0.63 \\
\hline AAS4 & 1.67 & 0.67 & 0.04 & 0.65 & 0.01 & 0.31 & 0.94 & 0.28 & 0.06 & 0.65 \\
\hline AAS5 & 1.69 & 0.68 & 0.04 & 0.66 & 0.01 & 0.30 & 0.95 & 0.29 & 0.05 & 0.67 \\
\hline AAS6 & 1.71 & 0.67 & 0.04 & 0.64 & 0.01 & 0.31 & 0.94 & 0.28 & 0.06 & 0.65 \\
\hline AAS7 & 1.67 & 0.68 & 0.04 & 0.65 & 0.01 & 0.30 & 0.94 & 0.29 & 0.06 & 0.66 \\
\hline AAS8 & 1.70 & 0.69 & 0.04 & 0.64 & 0.01 & 0.31 & 0.94 & 0.29 & 0.06 & 0.65 \\
\hline AAS9 & 1.69 & 0.68 & 0.04 & 0.67 & 0.01 & 0.28 & 0.94 & 0.29 & 0.06 & 0.68 \\
\hline AAS10 & 1.71 & 0.67 & 0.04 & 0.73 & 0.01 & 0.22 & 0.95 & 0.28 & 0.05 & 0.74 \\
\hline AAS11 & 1.70 & 0.65 & 0.05 & 0.78 & 0.01 & 0.17 & 0.95 & 0.28 & 0.06 & 0.79 \\
\hline AAS12 & 1.69 & 0.64 & 0.05 & 0.64 & 0.01 & 0.31 & 0.93 & 0.27 & 0.07 & 0.65 \\
\hline AAS13 & 1.71 & 0.65 & 0.05 & 0.79 & 0.01 & 0.15 & 0.95 & 0.28 & 0.05 & 0.80 \\
\hline AAS14 & 1.69 & 0.65 & 0.05 & 0.75 & 0.01 & 0.19 & 0.94 & 0.28 & 0.06 & 0.76 \\
\hline AAS15 & 1.70 & 0.65 & 0.05 & 0.80 & 0.01 & 0.15 & 0.95 & 0.28 & 0.05 & 0.81 \\
\hline AAS16 & 1.69 & 0.66 & 0.05 & 0.82 & 0.01 & 0.12 & 0.95 & 0.28 & 0.05 & 0.83 \\
\hline AAS17 & 1.74 & 0.66 & 0.05 & 0.79 & 0.01 & 0.16 & 0.95 & 0.28 & 0.06 & 0.80 \\
\hline AAS18 & 1.74 & 0.61 & 0.05 & 0.78 & 0.01 & 0.17 & 0.94 & 0.26 & 0.06 & 0.79 \\
\hline AAS19 & 1.74 & 0.63 & 0.05 & 0.78 & 0.01 & 0.16 & 0.94 & 0.27 & 0.06 & 0.79 \\
\hline AAS20 & 1.16 & 1.15 & 0.19 & 0.74 & 0.01 & 0.06 & 0.80 & 0.50 & 0.20 & 0.75 \\
\hline AAS21 & 1.18 & 1.14 & 0.19 & 0.77 & 0.01 & 0.03 & 0.80 & 0.49 & 0.20 & 0.78 \\
\hline AAS22 & 1.16 & 1.17 & 0.18 & 0.77 & 0.01 & 0.04 & 0.81 & 0.50 & 0.19 & 0.78 \\
\hline AAS23 & 1.16 & 1.16 & 0.18 & 0.78 & 0.01 & 0.02 & 0.81 & 0.50 & 0.19 & 0.80 \\
\hline AAS24 & 1.18 & 1.16 & 0.18 & 0.77 & 0.02 & 0.03 & 0.81 & 0.49 & 0.19 & 0.79 \\
\hline AAS25 & 1.16 & 1.15 & 0.17 & 0.68 & 0.01 & 0.14 & 0.80 & 0.50 & 0.20 & 0.69 \\
\hline AAS26 & 1.18 & 1.15 & 0.17 & 0.70 & 0.01 & 0.12 & 0.80 & 0.49 & 0.20 & 0.71 \\
\hline AAS27 & 1.48 & 0.59 & 0.07 & 0.57 & 0.01 & 0.35 & 0.89 & 0.28 & 0.11 & 0.58 \\
\hline AAS28 & 1.50 & 0.67 & 0.06 & 0.60 & 0.01 & 0.33 & 0.91 & 0.31 & 0.09 & 0.61 \\
\hline AAS29 & 1.46 & 0.67 & 0.06 & 0.59 & 0.01 & 0.35 & 0.91 & 0.31 & 0.09 & 0.60 \\
\hline AAS30 & 1.49 & 0.68 & 0.06 & 0.62 & 0.01 & 0.31 & 0.91 & 0.31 & 0.09 & 0.63 \\
\hline AAS31 & 1.55 & 0.71 & 0.07 & 0.65 & 0.01 & 0.28 & 0.91 & 0.31 & 0.09 & 0.66 \\
\hline AAS32 & 1.52 & 0.79 & 0.08 & 0.64 & 0.01 & 0.27 & 0.89 & 0.34 & 0.11 & 0.65 \\
\hline AAS33 & 1.54 & 0.74 & 0.08 & 0.63 & 0.01 & 0.29 & 0.89 & 0.32 & 0.11 & 0.64 \\
\hline \multicolumn{11}{|c|}{ Plage-Militaire beach } \\
\hline PMS1 & 1.30 & 1.03 & 0.07 & 0.67 & 0.01 & 0.33 & 0.56 & 0.44 & 0.09 & 0.68 \\
\hline PMS2 & 1.57 & 0.77 & 0.06 & 0.66 & 0.01 & 0.34 & 0.67 & 0.33 & 0.09 & 0.68 \\
\hline PMS3 & 1.91 & 0.26 & 0.06 & 0.68 & 0.01 & 0.32 & 0.88 & 0.12 & 0.08 & 0.69 \\
\hline PMS4 & 1.90 & 0.52 & 0.06 & 0.68 & 0.01 & 0.32 & 0.78 & 0.22 & 0.08 & 0.69 \\
\hline PMS5 & 1.91 & 0.26 & 0.06 & 0.68 & 0.01 & 0.32 & 0.88 & 0.12 & 0.08 & 0.69 \\
\hline PMS6 & 1.75 & 0.52 & 0.06 & 0.68 & 0.01 & 0.32 & 0.77 & 0.23 & 0.08 & 0.69 \\
\hline \multicolumn{11}{|c|}{ El Nasr beach } \\
\hline EN S1 & 0.69 & 1.88 & 0.16 & 0.68 & 0.00 & 0.16 & 0.56 & 0.73 & 0.19 & 0.69 \\
\hline EN S2 & 0.70 & 1.82 & 0.21 & 0.68 & 0.00 & 0.11 & 0.28 & 0.72 & 0.23 & 0.68 \\
\hline EN S3 & 0.70 & 1.92 & 0.15 & 0.68 & 0.01 & 0.17 & 0.27 & 0.73 & 0.18 & 0.68 \\
\hline EN S4 & 0.84 & 2.14 & 0.18 & 0.75 & 0.01 & 0.06 & 0.28 & 0.72 & 0.19 & 0.76 \\
\hline EN S5 & 0.69 & 2.30 & 0.13 & 0.74 & 0.00 & 0.13 & 0.23 & 0.77 & 0.15 & 0.74 \\
\hline EN S6 & 0.84 & 2.16 & 0.29 & 0.58 & 0.01 & 0.12 & 0.28 & 0.72 & 0.33 & 0.59 \\
\hline
\end{tabular}

$\mathrm{Xv}=\mathrm{X}$-vacancy, $\mathrm{Fe}^{*}(\mathrm{Y})=\mathrm{Y}(\mathrm{Fe} /(\mathrm{Fe}+\mathrm{Mg})), \mathrm{Mg}^{*}(\mathrm{Y})=\mathrm{Mg} /(\mathrm{Mg}+\mathrm{Fe}), \mathrm{Ca}^{*}(\mathrm{X})=\mathrm{X}(\mathrm{Ca} / \mathrm{Ca}+\mathrm{Na}), \mathrm{Na}^{*}=\mathrm{Na}+\mathrm{K}$. 


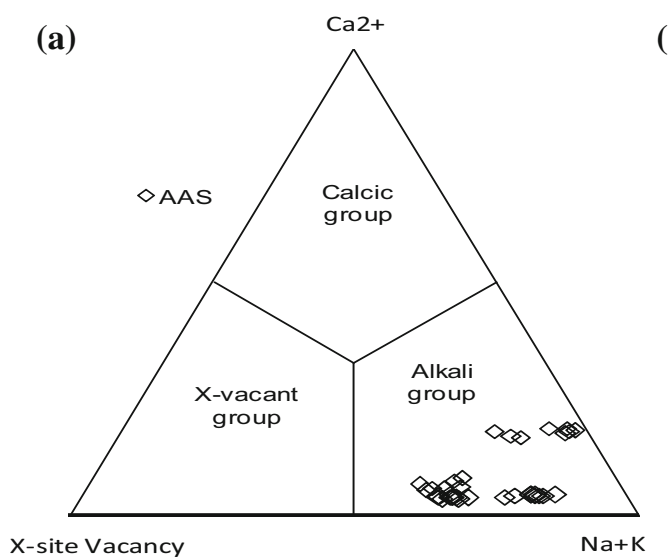

(b)

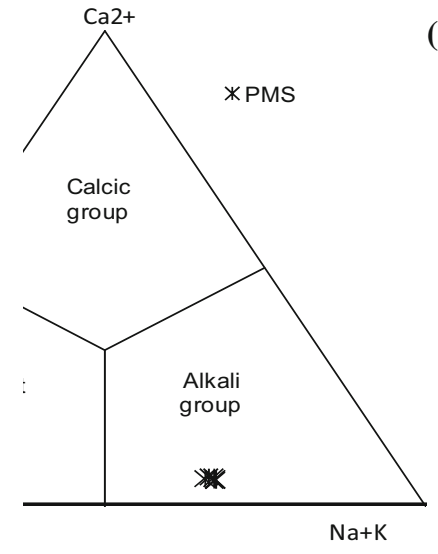

(c)

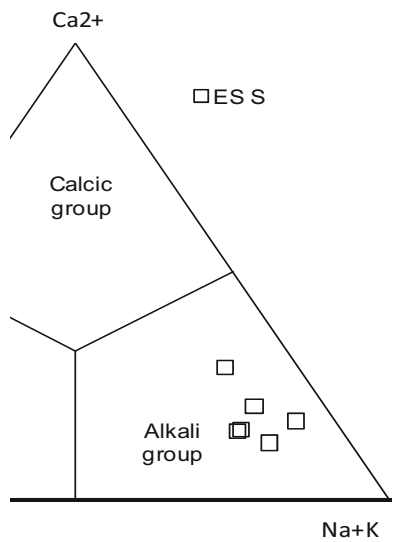

(d)

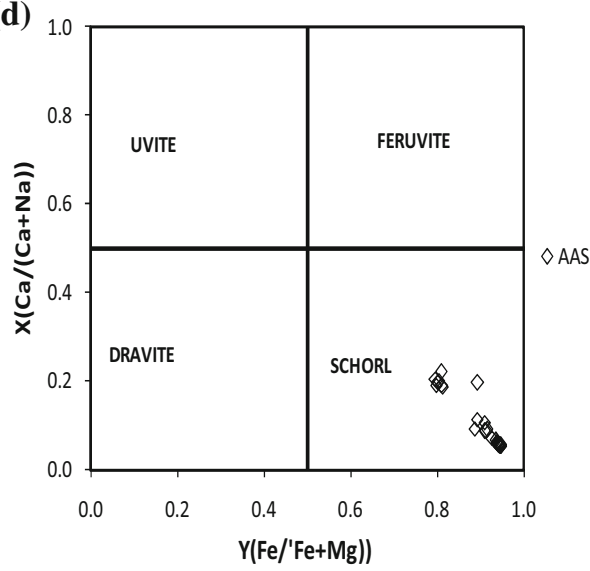

(e)

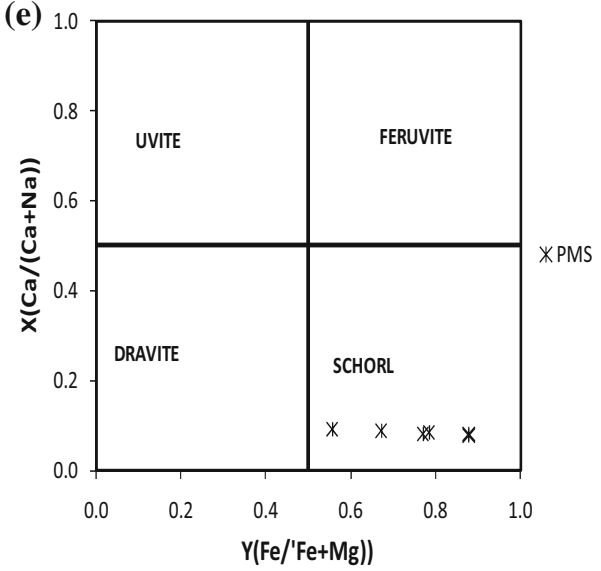

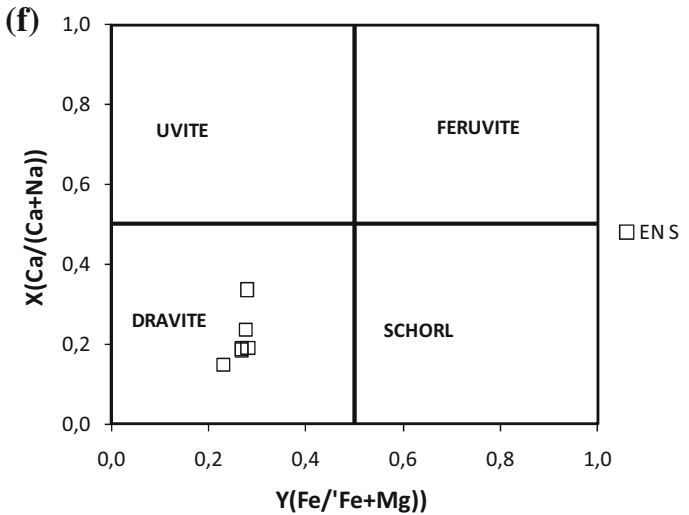

Figure 8. (a-c) Classification of the tourmaline from: (a) Ain Achir beach, (b) Plage-Militaire beach and (c) El Nasr beach in the ternary $\mathrm{Ca}^{2+}-\mathrm{X}$-site vacancy- $(\mathrm{Na}+\mathrm{K})$ system based on the dominant occupancy at the X-site; (d-f) Plot of tourmaline composition from: (d) Ain Achir beach, (e) Plage-Militaire beach, and (f) El Nasr beach in a $\mathrm{X}_{\mathrm{Fe}}^{\mathrm{Y}}$ vs. $\mathrm{X}_{\mathrm{Ca}}^{\mathrm{X}}$ plot (Jiang et al. 1995, 1996). AA S: Ain Achir sand, PM S: Plage-Militaire sand, EN S: El Nasr sand.

from the three studied beaches can be divided into three groups with different sources. Tourmalines from the Ain Achir beach are compositionally within field 2 of Li-poor granitoids and their associated pegmatites and partly in field 4 of Ca-poor metapelites, metapsammites and quartz-tourmaline rocks (figure 10a) suggesting a mixed source rock for these tourmalines (e.g., tourmaline-rich quartzo-feldspathic veins in the garnet-staurolite micaschists and in the garnet micaschists or a single source with two tourmaline types being more or less Fe-rich). All the tourmaline samples from the Plage-Militaire beach plot in the Li-poor granitoids and their associated pegmatites field 2 (figure 10b) indicating a single source (tourmaline-rich 


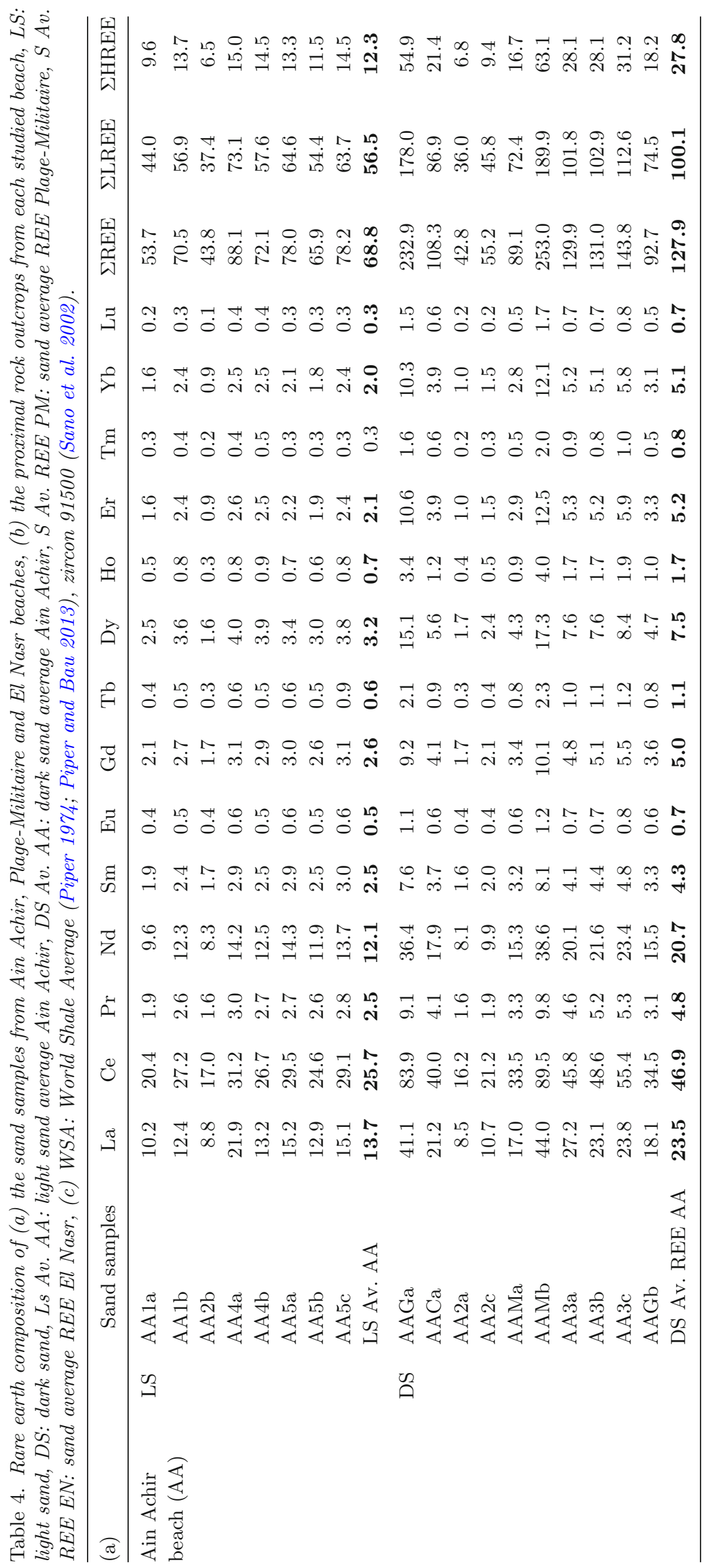




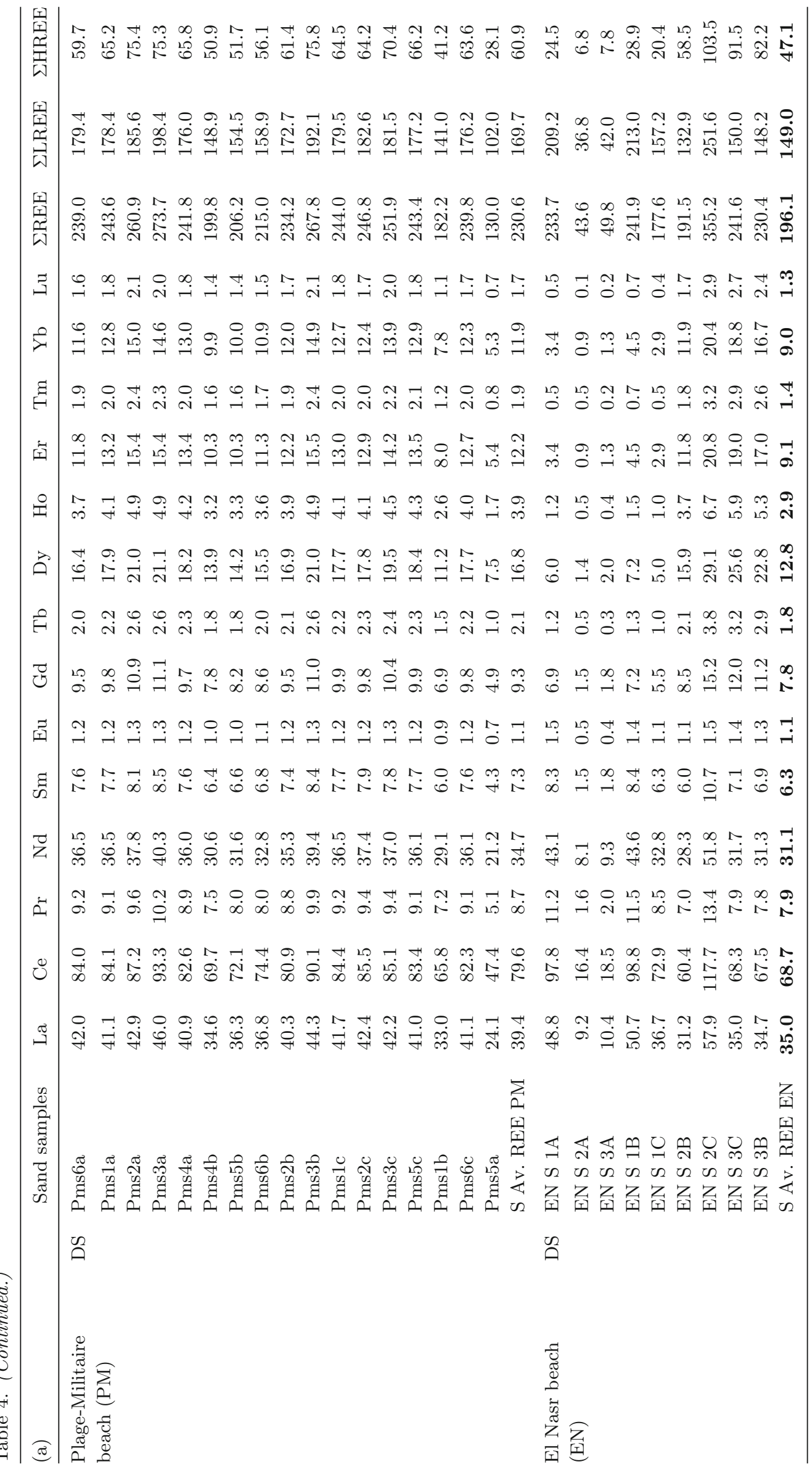




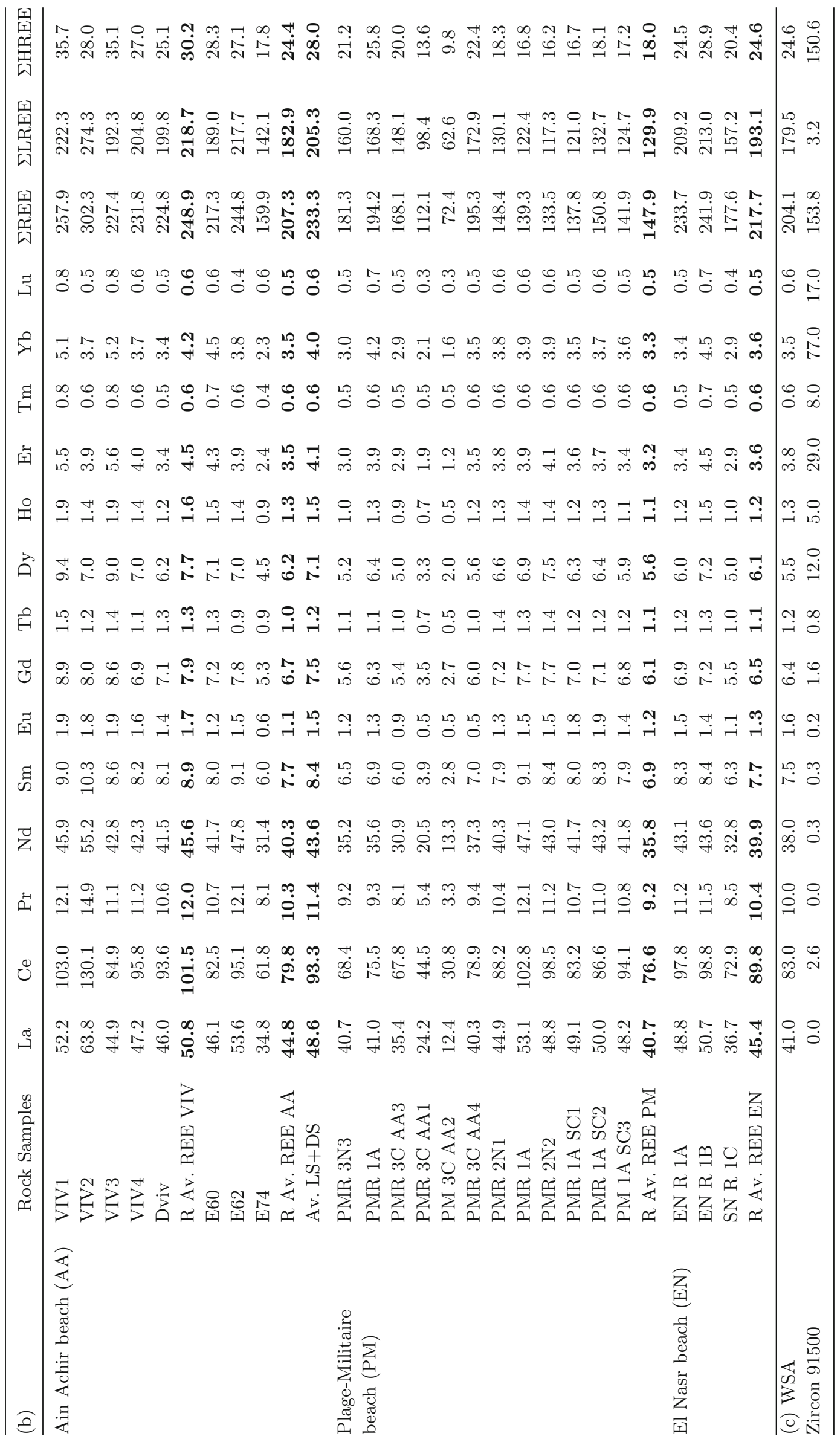



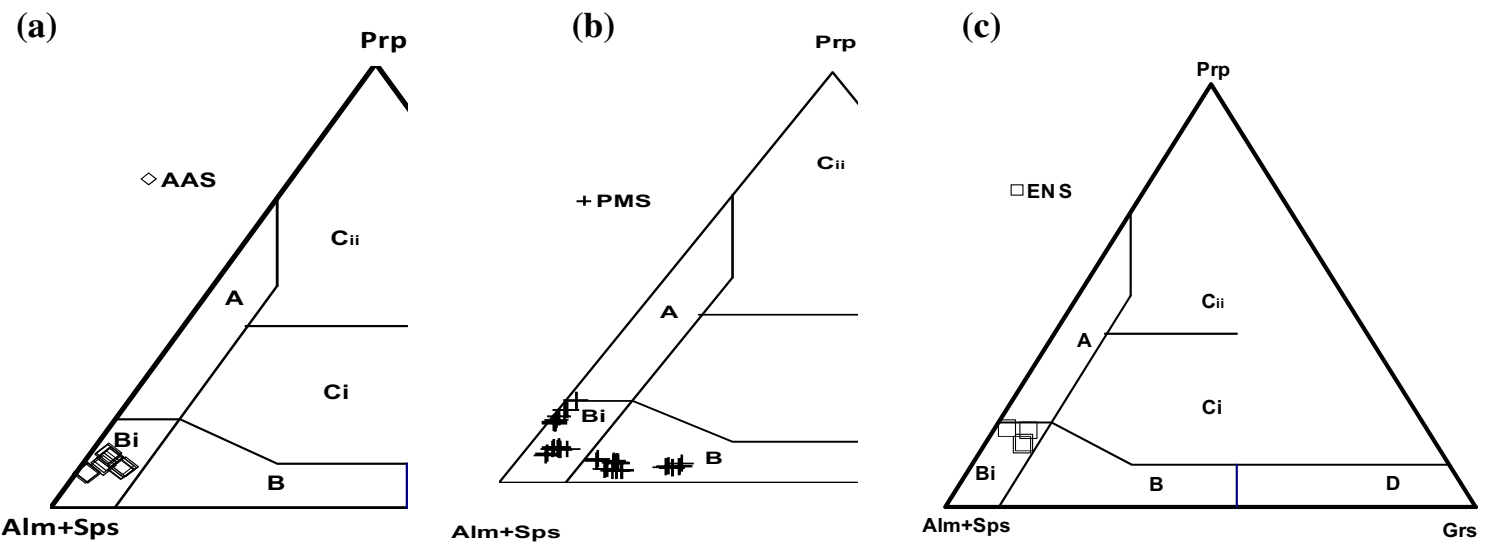

Figure 9. The composition of garnets in the ternary classification diagram of Mange and Morton (2007) with almandine+spessartine, grossular and pyrope as poles: (a) Ain Achir, (b) Plage-Militaire, (c) El Nasr sands. A - high-grade granulite-facies metasedimentary rocks and intermediate felsic igneous rocks; B - amphibolite-facies metasedimentary rocks; $\mathrm{Bi}$ - intermediate to felsic igneous rocks; $\mathrm{Ci}$ - mainly high-grade mafic rocks; Cii - ultramafics with high $\mathrm{Mg}$ (pyroxenites and peridotites); D - metasomatic rocks, very low-grade metamafic rocks and ultrahigh-temperature metamorphosed calc-silicate granulites. AA S: Ain Achir sand, PM S: Plage-Militaire sand, EN S: El Nasr sand.
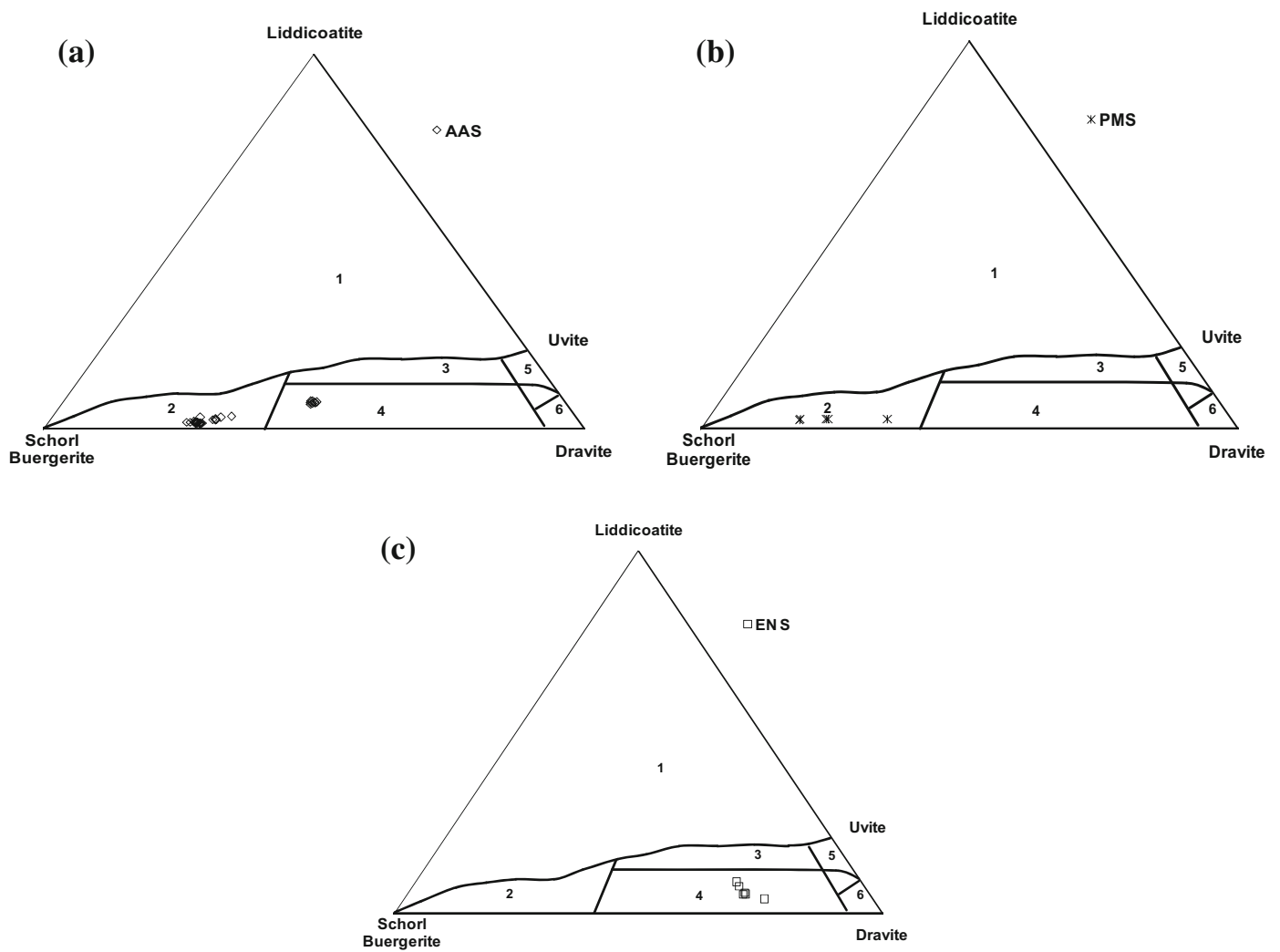

Figure 10. Microprobe analyses of tourmaline samples plotted on $\mathrm{Ca}-\mathrm{Fe}^{(\mathrm{t})}-\mathrm{Mg}$ diagram of Henry and Guidotti (1985): (a) Ain Achir, (b) Plage-Militaire, (c) El Nasr samples. 1. Li-rich granitoid, pegmatites and aplites; 2. Li-poor granitoids and their associated pegmatites; 3. Ca-rich metapelites and metapsammites and calc-silicate rocks; 4. Ca-poor metapelites, metapsammites and quartz-tourmaline rocks; 5. metacarbonates; 6. metaultramafics. AA S: Ain Achir sand, PM S: PlageMilitaire sand, EN S: El Nasr sand.

quartzo-feldspathic veins, e.g., in the garnet micaschists). In contrast to the two abovementioned beaches, tourmalines from the El Nasr cluster in field 4 corresponding to Ca-poor metapelites, metapsammites and quartz-tourmaline rocks (figure 10c). The source rock from the El Nasr tourmaline is different from those from the Ain Achir and Plage-Militaire beaches. 


\subsection{The rare earth element behaviour of whole sand and rock}

In order to identify the provenance, the REE data from the sand samples are compared to data from the rock outcrops located relatively close to the studied sand beaches. All samples (sand and rock) from the studied areas have been normalised to the World Shale Average (WSA; Piper 1974; Piper and Bau 2013).

Many authors (e.g., Jung et al. 2006, 2012; Leybourne and Johannesson 2008) show that fractionation factors $\mathrm{LREE}_{\mathrm{N}} / \mathrm{HREE}_{\mathrm{N}}$ and $\mathrm{MREE}_{\mathrm{N}} /$ HREE $_{\mathrm{N}}$ (LREEN: La ${ }_{\mathrm{N}}+\operatorname{Pr}_{\mathrm{N}}+\mathrm{Nd}_{\mathrm{N}} ;$ MREE $_{\mathrm{N}}$ : $\left.\mathrm{Sm}_{\mathrm{N}}+\mathrm{Eu}_{\mathrm{N}}+\mathrm{Tb}_{\mathrm{N}} ; \mathrm{HREE}_{\mathrm{N}}: \mathrm{Ho}_{\mathrm{N}}+\mathrm{Yb}_{\mathrm{N}}+\mathrm{Lu}_{\mathrm{N}}\right)$ are helpful to discriminate among source rocks.

The fractionation factors $\left(\mathrm{LREE}_{\mathrm{N}} / \mathrm{HREE}_{\mathrm{N}}\right.$ and $\mathrm{MREE}_{\mathrm{N}} / \mathrm{HREE}_{\mathrm{N}}$ ) of the sand samples from each beach have been plotted on the mixing diagram and compared with the rock samples (figure 11). In Ain Achir, light and the dark sand fractionation factors overlap with most of the Plage-Militaire rock outcrops (figure 11a) suggesting that the latter could be their provenance source. The Plage-Militaire sand samples plot close to/or overlap with the adjacent basement rock (Plage-Militaire) and distal (El Nasr) outcrops suggesting mixed sources of the sand (figure 11b). At the El Nasr beach the sand samples have a weak link to the outcrops in their vicinity (in view distance), a portion of the sand samples plot close to the data from the distal Cap de Garde kyanite-garnet micaschists (R Vivier) and staurolite-garnet micaschists (R Ain Achir), suggesting that these two kinds of rocks (which are probably outcropping at sea, close to the El Nasr beach) could be the source of the sand (figure 11c).

On the normalised to the WSA (Piper 1974; Piper and Bau 2013) spider-gram (figure 12a) all costal rocks display very similar flat REE patterns suggesting that these rocks have similar geological and geochemical backgrounds (figure 12a). Compared to their associated rocks and to those from the Plage-Militaire and El Nasr beaches, some Ain Achir beach sand REE patterns are more depleted. Some others, mostly dark sand samples, have similar depleted LREE and enriched HREE patterns (figure $12 \mathrm{~b}-\mathrm{d}$ ) with respect to the rocks, point to the influence of a HEE-rich mineral (e.g., zircon). Zircon is the main mineral in the majority of igneous and metamorphic rocks. It is a host for
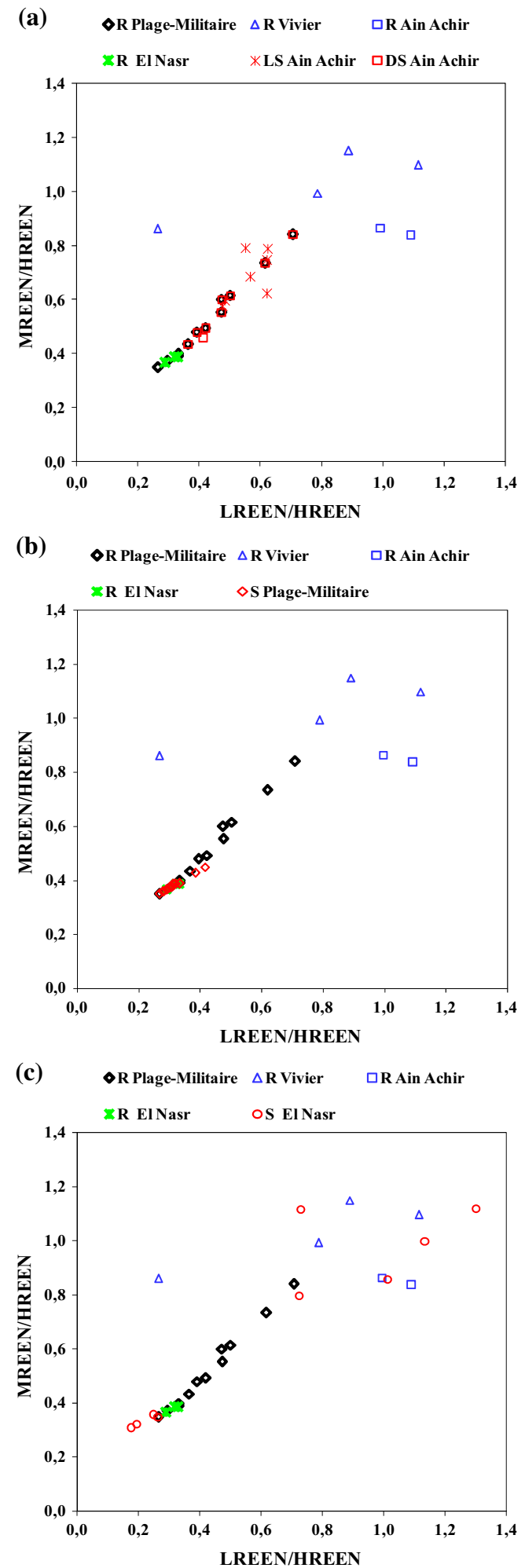

Figure 11. Fractionation factors diagram between $\mathrm{LREE}_{\mathrm{N}} / \mathrm{HREE}_{\mathrm{N}}$ and $\mathrm{MREE}_{\mathrm{N}} / \mathrm{HREE}_{\mathrm{N}}$ (LREEN: La $\mathrm{La}_{\mathrm{N}}+$ $\operatorname{Pr}_{\mathrm{N}}+\mathrm{Nd}_{\mathrm{N}} ; \mathrm{MREE}_{\mathrm{N}}: \mathrm{Sm}_{\mathrm{N}}+\mathrm{Eu}_{\mathrm{N}}+\mathrm{Tb}_{\mathrm{N}} ; \mathrm{HREE}_{\mathrm{N}}:$ $\left.\mathrm{Ho}_{\mathrm{N}}+\mathrm{Yb}_{\mathrm{N}}+\mathrm{Lu}_{\mathrm{N}}\right)$ for the sand and all rock samples of $(\mathbf{a})$ Ain Achir beach, (b) Plage-Militaire beach and (c) El Nasr beach. R: rock, LS: light sand, DS: dark sand. 
(a)

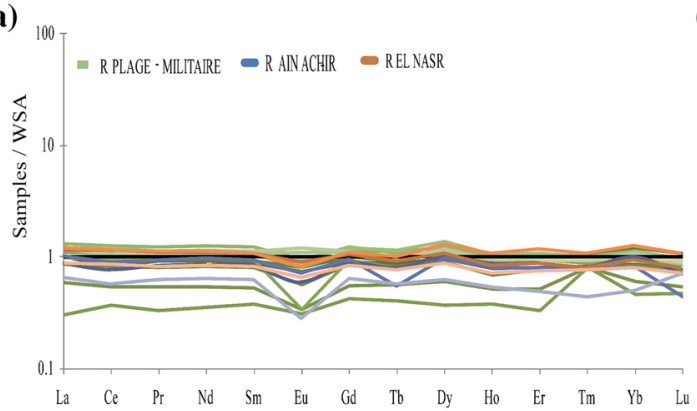

(c)

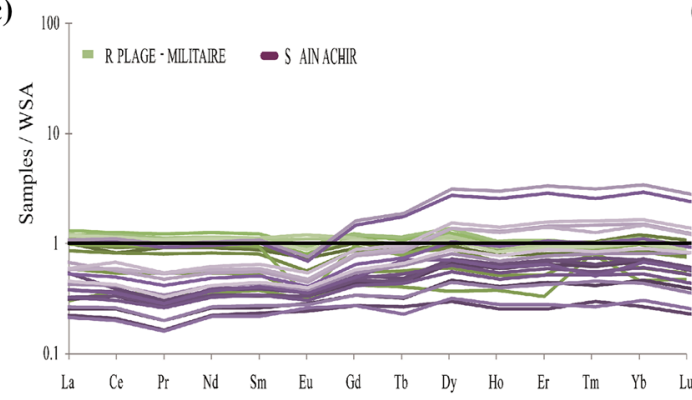

(e)

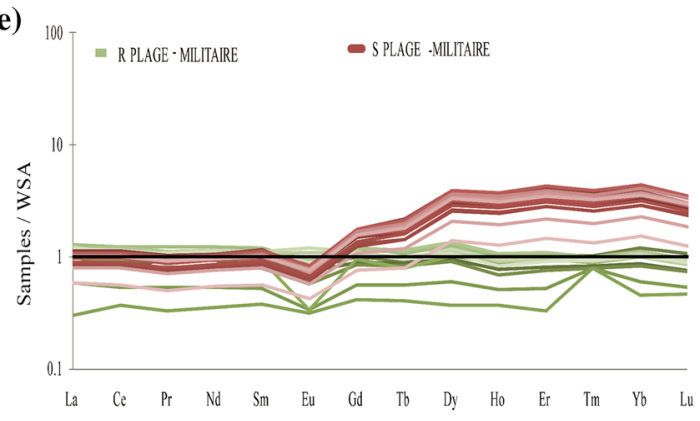

(g)

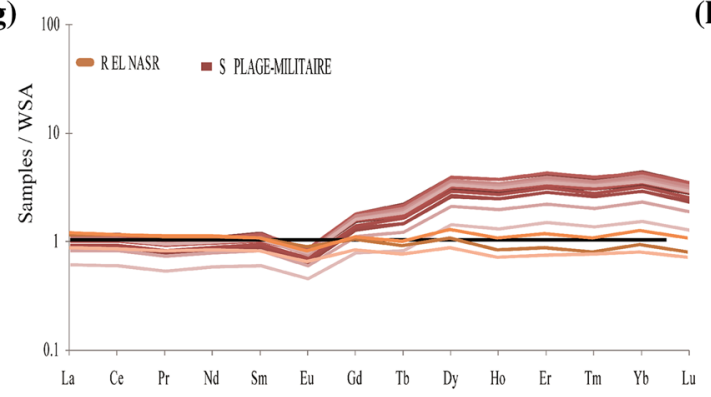

(i) 100

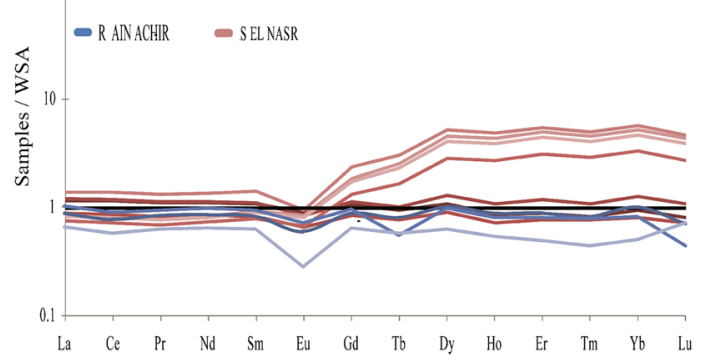

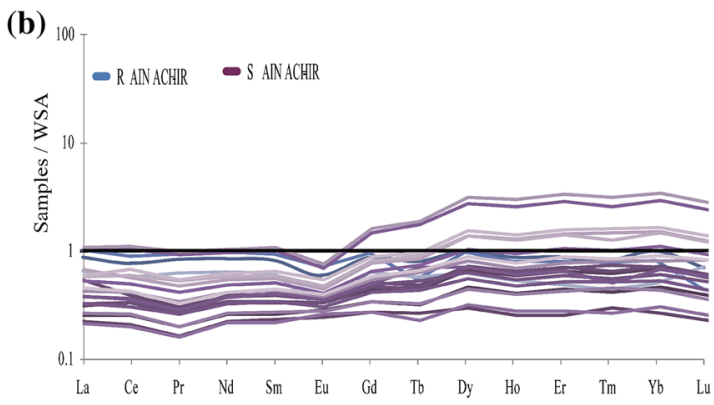

(d)

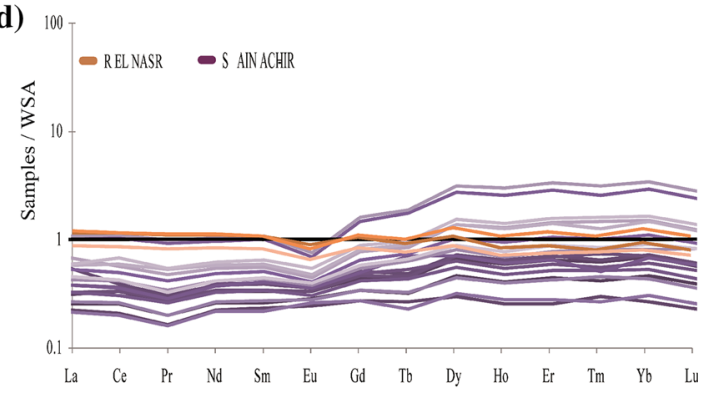

(f)

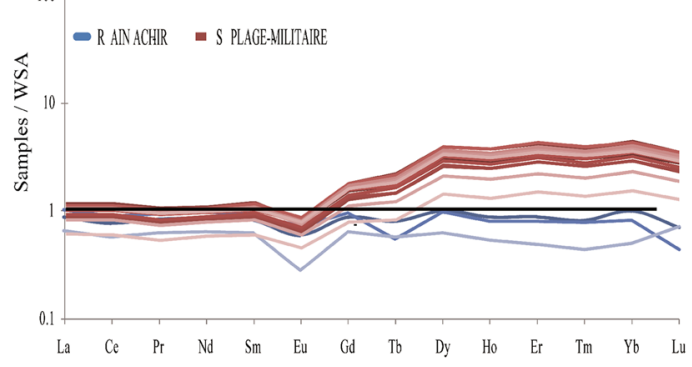

(h)

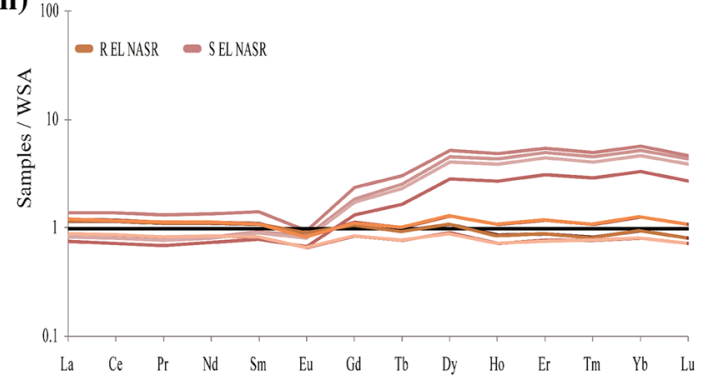

(j)

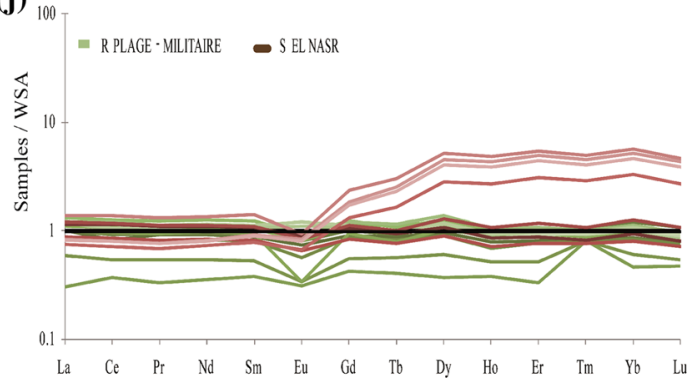

Figure 12. WSA-normalised rare earth element pattern of (a) rock samples from all three beaches, (b) sand and rocks from the Ain Achir beach, (c) Ain Achir sand and rocks from the Plage-Militaire beach, (d) Ain Achir sand and rocks from the El Nasr beach, (e) sand and rocks from the Plage-Militaire beach, (f) Plage-Militaire sand and rocks from the Ain Achir beach, (g) Plage-Militaire sand and rocks from the El Nasr beach, (h) sand and rocks from the El Nasr beach, (i) El Nasr sand and rocks from the Ain Achir beach, and (g) El Nasr sands and rocks from the Plage-Militaire beach. 

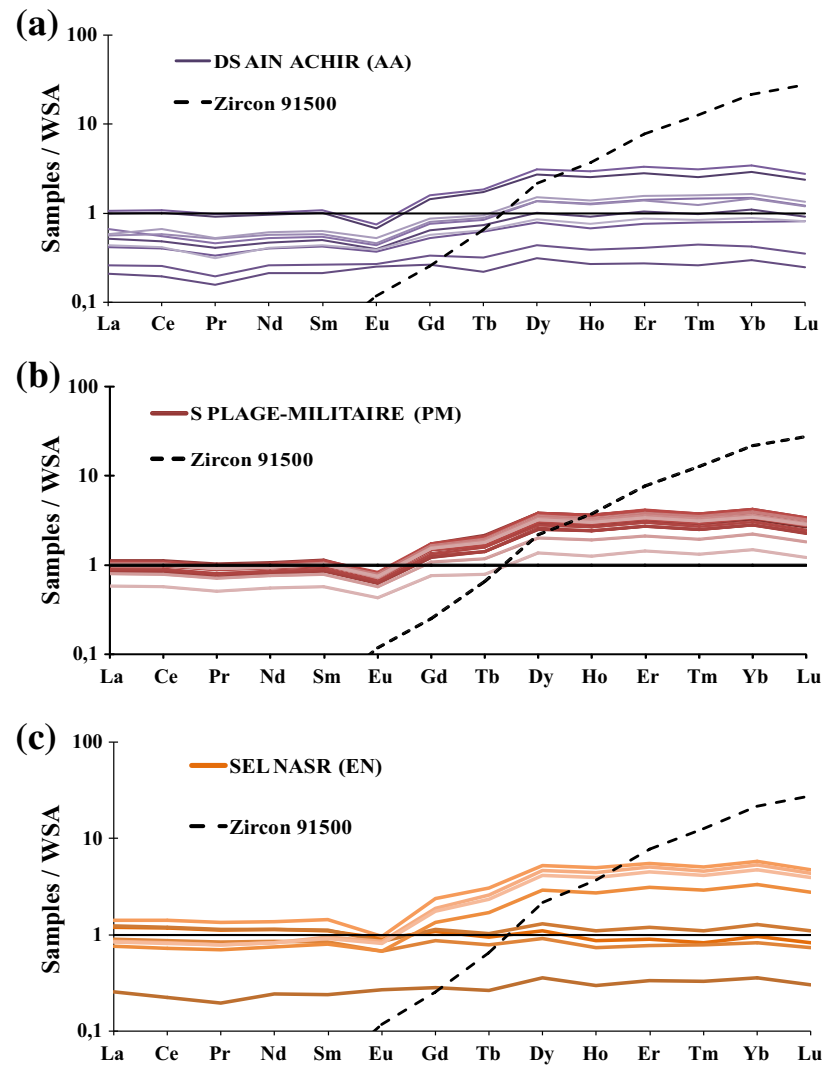

Figure 13. WSA-normalised rare earth element pattern of the standard zircon 91500 (Sano et al. 2002) compared with (a) Ain Achir dark sand, (b) Plage-Militaire sand, and (c) El Nasr beach sand.

significant fractions of the whole-rock abundance of the REE (Bea and Montero 1999; O'Hara et al. 2001). All Plage-Militaire sand samples display different WSA-normalised (Piper 1974; Piper and Bau 2013) REE patterns with their proximal rock outcrops and with those from the Ain Achir (figure 12e and f) but their REE distribution is quite similar to those from the El Nasr rocks (figure 12g). Therefore, it can be concluded that the Plage-Militaire sand has a mixed source. A portion of the Plage-Militaire sand could be from the proximal rock outcrops, which are mainly staurolite-garnet micaschists and from formations similar to those of the El Nasr beach (garnet-staurolite micaschists). REEs spider graphs of El Nasr sands show differences with respect to their closeby rocks (figure 12h) and also to Ain Achir (figure 12i) and the PlageMilitaire (figure 12j) rocks. They show a flat LREE pattern and are enriched in HREE, suggesting a different metamorphic source with a HREErich mineral composition. All WSA-normalised sand samples show negative europium anomalies. Some studies suggest that they are due to non-selective losses of REE during weathering and that Eu-anomalies are inherited from the sediment source (Nesbitt 1979; Condie 1991; Condie et al. 1995; Nesbitt and Young 1996).

Despite that, the Ain Achir, Plage-Militaire and El Nasr sand samples have nearly the same mineralogical compositions; some sand samples show a stronger HREEs enrichment than others. This is indicative of influence by a HEE-rich mineral. XRD analyses of staurolites from these sand samples show that their inclusions are mostly zircon grains. The comparison of WSA-normalised REE (Piper 1974; Piper and Bau 2013) pattern of sand samples from the Ain Achir, Plage-Militaire and El Nasr sands with selected zircon from the literature (Standard 91500, Sano et al. 2002) shows similar HREE pattern (figure 13). The possibility of zircon geochemistry influencing the HREEs distribution cannot be excluded.

\section{Conclusion}

Because of their flat topography and the lack of an active hydrographic system, the Cap de Garde (Ain Achir and Plage-Militaire beaches) and the Harbor (El Nasr beach) fields are characterised by insignificant low continental erosion processes. The seasonal swell and sea wave (1-3 m) are the principal erosion factors and beach sedimentation along the coast. They contribute to the beach and near-shore sand accumulation. The heavy-sand buildup is due to a hydraulic (sea water) segregation process. The mineral assemblages (staurolite, garnet, tourmaline, kyanite and rare epidote) of the three heavy-sand beaches are similar and point to the influence of metamorphic rocks as source areas and reflect the properties of these sources. The compositions of detrital garnet and tourmaline in the heavy-mineral sands vary according to the beach location. The Ain Achir sand is characterised by extreme almandine-rich garnet and schorl-tourmaline. The Plage-Militaire heavysand shows two kinds of garnets: Fe-rich almandine and $\mathrm{Mg}$-rich almandine. Their tourmaline is schorl rich in composition. The high $\mathrm{Mg}$ concentration in some garnets and tourmaline-rich sand samples from the El Nasr beach reflect a magnesian-rich source.

Compared to their associated rock outcrops, the WSA-normalised REE pattern of the dark sand samples from Ain Achir, Plage-Militaire and El Nasr beaches show similar LREE patterns and enrichment of HREE suggesting the influence of 
a HEE-rich mineral (e.g., zircon as inclusions in staurolite). Ain Achir sands reveal a mixed provenance: from their proximal rock outcrops garnet-staurolite micaschist (Ain Achir and Plage Militaire) and rocks similar to garnet-staurolite micaschists at the El Nasr beach outcrops. The Plage-Militaire sand samples display different normalised WSA REE patterns as compared to their proximal rock outcrops and to those from the Ain Achir. But looks quite similar to those from the El Nasr rock formations (garnet-staurolite micaschist). Sand REE distribution curves of the El Nasr show differences when compared to those from the rocks in the vicinity to Ain Achir and to the Plage-Militaire outcrops suggesting a different metamorphic source area with an HREE-rich mineral composition.

The source area of kyanite in the Ain Achir sand could be the proximal staurolite-garnet kyanite micaschists at the Plage-Militaire. The source rocks of the epidote in the sand of Ain Achir and the Plage Militaire are the epidote-bearing skarns.

The present study shows a complex mixture in the of beach sediments, although the sources seem to be very close and easily determinable. The results from the unsolidified modern beach sand could help to evaluate the sedimentation process and provenance of solidified layers. In provenance studies of fossil beach sediment, the careful study of related outcrops can help to evaluate different sources, proximal as well as distal. A proximal situation is characterised by the similarity of mineral assemblage and proportions in different outcrops. A deviation from this points to distal sedimentation. In addition, the results add important aspects for provenance analyses of fossil heavy-mineral placer deposits, where the source evaluation is an important economic factor.

\section{Acknowledgements}

We are very grateful to anonymous reviewers for their helpful comments and suggestions that improved this paper. Moreover, we thank the Institute of Earth and Environmental Science, the University of Potsdam for their support in analytical facilities.

\section{References}

Ahmed-Said Y, Leake B E and Rogers G 1993 The petrology, geochemistry and petrogenesis of the Edough igneous rocks, Annaba, NE Algeria; J. Afr. Earth Sci. 17(1) 111-123.

ANDI 2013 (Agence Nationale de développement de l'Investissement) Invest in Algeria, Wilaya de Annaba.

Bea F and Montero P 1999 Behaviour of accessory phases and redistribution of $\mathrm{Zr}, \mathrm{REE}, \mathrm{Y}$, Th and $\mathrm{U}$ during metamorphism and partial melting of metapelites in the lower crust: An example from the Kinzigite Formation of Ivrea-Verbano, NW Italy; Geochim. Cosmochim. Acta 63 1133-1153.

Blatt H, Tracy R J and Owens E 2006 Petrology: Igneous, sedimentary and metamorphic, 3rd edn, W.H. Freeman and Co, New York.

Brunnel M, Hammor D, Misseri M, Gleizes G and Bouleton J 1988 Cisaillements synmétamorphes avec transport vers le Nord-Ouest dans le massif cristallin de l'Edough (Est Algérien); C.R. Acad. Sci. 306 1039-1045.

Caby R and Hammor D 1992 Le Massif cristallin de l'Edough (Algérie): Un "Métamorphic Core Complex" d'âge miocène dans les Magrébides; C.R. Acad. Sci. 314 829-835.

Caby R, Hammor D and Delor C 2001 Metamorphic evolution, partial melting and Miocene exhumation of lower crust in the Edough metamorphic core complex, west Mediterranean orogen, eastern Algeria; Tectonophysics 342 239-273.

Condie K C 1991 Another look at rare earth elements in shales; Geochim. Cosmochim. Acta 55 2527-2531.

Condie K C, Dengate J and Cullers R L 1995 Behavior of rare elements in a paleo-weathering profile on granodiorite in the Front Range, Colorado, USA; Geochim. Cosmochim. Acta 59 279-294.

Darnley A G, Bjorklund A, Bolviken B, Gustavsson N, Koval P V, Plant J A, Steenfel A, Tauchid M and Xie X 1995 A Global geochemical database for environmental and resource management. Recommendations for international geochemical mapping; Final Report of IGCP Project 259, UNESCO Publishing.

Henry D J and Guidotti C V 1985 Tourmaline as a petrogenetic indicator mineral: An example from the staurolitegrade metapelites of NW Maine; Am. Miner. 70 1-15.

Henry D J, Novák M, Hawthorne F C, Ertl A, Dutrow B L, Uher P and Pezozotta F 2011 Nomenclature of the tourmaline-supergroup minerals; Am. Miner. 96 895-913.

Hilly J 1962 Etude geologique du massif de l'Edough et du Cap de Fer (Est-Constantinois); Pub. Serv. de la Carte Géol. Algérie 19 1-408.

http://www.temperatureweather.com/mediterr/meteo/frmeteo-en-algerie-annaba.html

http://fr.wisuki.com/statistics/4809/annaba?a_wi=4\&wi_m $=0 \& a_{-}$wa $=0 \&$ wa_m $=1 \&$ temp $=$ monthly\&rain=quantity.

Hufty A 2001 Introduction à la climatologie, Les presses de l'Université de Laval, De Böeck Université, 545.

Jiang S Y, Palmer M R, Li Y H and Xue C J 1995 Chemical compositions of tourmaline in the YindongziTongmugou $\mathrm{Pb}-\mathrm{Zn}$ deposits, Qinling, China: Implications for hydrothermal ore-forming processes; Miner. Depos. 30 225-234.

Jiang S Y, Palmer M R, McDonald A M, Slack J F and Leitch C H B 1996 Feruvite from the Sullivan Pb-Zn-Ag deposit, British Columbia; Can. Mineral. 34 733-740. 
Jung H S, Lim D I, Yang S Y and Yoo H S 2006 Constraints of REE distribution patterns in core sediments and their provenance, Northern East China; Econ. Env. Geol. Sea 39(1) 39-51 (in Korean with English abstract).

Jung H S, Lim D, Choi J Y, Yoo H S, Rho K C and Lee H B 2012 Rare earth element compositions of core sediments from the shelf of the South Sea, Korea: Their controls and origins; Cont. Shelf Res. 48 75-86.

Leybourne M I and Johannesson K H 2008 Rare earth elements (REE) and yttrium in stream waters, stream sediments, and Fe-Mn oxyhydroxides: Fractionation, speciation, and controls over REE + Y patterns in the surface environment; Geochim. Cosmochim. Acta 72 5962-5983.

Locock A J 2008 An Excel spreadsheet to recast analyses of garnet into end-member components, and a synopsis of the crystal chemistry of natural silicate garnets; Comput. Geosci. 34 1769-1780.

Mange M A and Maurer H F W 1991 Schwerminerale in Farbe; Stuttgart, Enke, 148p.

Mange M A and Wright D T (eds) 2007 Heavy minerals in use; Elsevier, Amsterdam, 1283p.

Mesurim: http://acces.ens-lyon.fr/acces/logiciels/applicatio ns/mesurim

Morton A C and Hallsworth C 1994 Identifying provenancespecific features of detrital heavy mineral assemblages in sandstones; Sedim. Geol. 90 241-256.

Nesbitt H W 1979 Mobility and fractionation of rare earth elements during weathering of a granodiorite; Nature $\mathbf{2 7 9}$ 206-210.

Corresponding editor: PARTha PRATim Chakraborty
Nesbitt H W and Young G W 1996 Petrogenesis of sediment in the absence of chemical weathering effects of abrasion and sorting on bulk composition and mineralogy; Sedimentology 43 341-358.

O'Hara M J, Fry N and Prichard H M 2001 Minor phases as carriers of trace elements in non-modal crystal-liquid separation processes II: Illustrations and bearing on behaviour of REE, U, Th and the PGE in igneous processes; $J$. Petrol. 42 1887-1910.

Oularbi A and Zeghiche A 2009 Sensibilité à l'érosion du massif cristallophyllien de l'Edough (Nord-Est Algérien); Synthèse 20 58-72.

Piper D Z 1974 Rare earths in the sedimentary cycle: A summary; Chem. Geol. 4 285-304.

Piper D Z and Bau M 2013 Normalized rare earth elements in water, sediments, and wine: Identifying sources and environmental redox conditions; Am. J. Analyt. Chem. 4(10A) 69-83.

Sano Y, Terada K and Fukuoka T 2002 High mass resolution ion microprobe analysis of rare earth elements in silicate glass, apatite and zircon: Lack of matrix dependency; Chem. Geol. 184 217-230.

Whitney D L and Evans B W 2010 Abbreviations for names of rock-forming minerals; Am. Miner. 95(1) 185-187.

Yavuz F, Yavuz V and Sasmaz A 2006 WinClastour A visual basic program for tourmaline formula recalculation and classification; Comput. Geosci. 32 $1156-1168$ 